\title{
A Survey on Fingerprint Minutiae-based Local Matching for Verification and Identification: Taxonomy and Experimental Evaluation
}

\author{
Daniel Peralta ${ }^{\mathrm{a}}$, Mikel Galar ${ }^{\mathrm{c}}$, Isaac Triguero ${ }^{\mathrm{d}, \mathrm{a}}$, Daniel Paternain ${ }^{\mathrm{c}}$, Salvador García ${ }^{\mathrm{a}, \mathrm{b}, *}$, \\ Edurne Barrenechea ${ }^{c}$, José M. Benítez ${ }^{a}$, Humberto Bustince ${ }^{c}$, Francisco Herrera ${ }^{a}$ \\ ${ }^{a}$ Dept. of Computer Science and Artificial Intelligence. University of Granada, 18071 Granada, Spain \\ ${ }^{b}$ Faculty of Computing and Information Technology - North Jeddah, King Abdulaziz University, 21589, \\ Jeddah, Saudi Arabia \\ ${ }^{c}$ Departamento de Automática y Computación, Universidad Pública de Navarra, Pamplona, Spain \\ ${ }^{d}$ Inflammation Research Center, a VIB-UGent Dept. UGent Dept. of Internal Medicine, Respiratory \\ Medicine (GE01) Technologiepark 927, B-9052 Zwijnaarde, Belgium.
}

\begin{abstract}
Fingerprint recognition has found a reliable application for verification or identification of people in biometrics. Globally, fingerprints can be viewed as valuable traits due to several perceptions observed by the experts; such as the distinctiveness and the permanence on humans and the performance in real applications. Among the main stages of fingerprint recognition, the automated matching phase has received much attention from the early years up to nowadays. This paper is devoted to review and categorize the vast number of fingerprint matching methods proposed in the specialized literature. In particular, we focus on local minutiae-based matching algorithms, which provide good performance with an excellent trade-off between efficacy and efficiency. We identify the main properties and differences of existing methods. Then, we include an experimental evaluation involving the most representative local minutiae-based matching models in both verification and evaluation tasks. The results obtained will be discussed in detail, supporting the description of future directions.
\end{abstract}

Keywords: Biometrics, fingerprint verification, fingerprint identification, local matching, minutiae.

*Corresponding author. Tel.: +34 958 240598; fax: +34 958243317.

Email addresses: dperalta@decsai.ugr.es (Daniel Peralta), mikel.galar@unavarra.es (Mikel Galar), Isaac.Triguero@irc.vib-UGent.be (Isaac Triguero), daniel.paternain@unavarra.es (Daniel Paternain), salvagl@decsai.ugr.es (Salvador García), edurne.barrenechea@unavarra.es (Edurne Barrenechea), J.M.Benitez@decsai.ugr.es (José M. Benítez), bustince@unavarra.es (Humberto Bustince), herrera@decsai.ugr.es (Francisco Herrera) 


\section{Introduction}

Automatic fingerprint recognition has been one of the most known and used biometric authentication systems during the last decades. It has been used for personal verification and identification with great achievements [76]. A vast number of applications incorporate fingerprint recognition as basics, such as forensics, building accessing, ATM authentication or secure payment [113]. There are some other human characteristics that can be used as traits of a biometric system, such as the person's face, the retina or iris [16], the voice, etc. There is no trait that highlights as the best one. However, on average, fingerprints offer good capabilities in all properties analyzed by the experts and excellent results in distinctiveness [126], permanence and global performance [113]. Although the recognition is not as accurate as with other traits, it provides a good balance between accuracy, speed, resource requirements and robustness.

Independent of the type of task, either verification [72] (one-to-one comparison) or identification (search for an input fingerprint in a database) [80], it is necessary to perform a sequence of operations to build a template database and later use the system. Assuming that there is a database and that proper enrollments have been previously taken, the order of the operations for both tasks is given by: a capture of the fingerprint, a feature extraction stage, a matching and a pre-selection or filtering [85] (which is associated to identification tasks only). The capture of the fingerprint obtains an image that is not usually stored as such in the database. Instead, a feature extraction process is applied to obtain up to three levels of features [60]: level 1 features provide, at the global level, information of singular points and ridge line flow or orientation; level 2 features, at a local level, refer to minutiae details which usually correspond to bifurcations and ridge endings; and level 3 features, at the very-fine level, include features inside the ridges such as width, shape, curvature, dots, etc. These features are only observable in high resolution images.

Once a set of features is extracted from the fingerprint image, the final goal is to find (or confirm) the identity of a person whose fingerprint has been previously enrolled into the system. The matching mechanism is the responsible to provide a likeliness score between two fingerprints. Most of the efforts in matching are with the use of minutiae details, although there are other types of matching methods based on correlations of images, other types of features and even on level 3 features. Minutiae matching consists of finding the alignment between two templates that results in the maximum number of minutiae pairings. Furthermore, minutiae matching can be classified as local or global [81], aligned or not [189], etc; all the categories will be detailed in this paper.

Many fingerprint matching algorithms have been proposed in the literature, and the operations with features they use are sometimes similar or even repeated. In spite of the existence of some reviews on the topic, such as $[174,113,71]$, they are not explicitly focused on matching and the characteristics of the methods are not completely studied or categorized. This issue may lead to a lack of unification and even to propose very similar matching methods in the future. Moreover, there are few attempts to empirically compare them.

In this sense, the motivation of this paper can be segregated into three main objectives:

- To gather and briefly describe all the matching methods proposed in the specialized 
literature.

- To offer an entire taxonomy based on the main processes and properties observed in the matching methods. It allows us to understand the reasons to choose the most suitable matching algorithm depending on the circumstances.

- To conduct an empirical study analyzing the most important local minutiae-based matching algorithms in terms of accuracy and speed throughput when they are applied to both verification and identification tasks.

The rest of this paper is organized as follows. Section 2 provides the necessary background in fingerprint minutiae matching. In Section 3, we introduce the main properties and the taxonomy for the matching methods. Next, Section 4 overviews the current trends in fingerprint matching. In Section 5, experiments on several data sets compare some of the most important local minutiae-based matching methods. Finally, Section 6 concludes the paper, including some original opinions for instruction in theory and application and future research directions. Additional material to the paper can be found at http://sci2s.ugr.es/MatchingReview/.

\section{Background in Fingerprint Minutiae Matching}

Fingerprint matching is a crucial step in both verification and identification problems. Roughly, a fingerprint matching algorithm compares two fingerprints and returns either a degree of similarity (a real number bounded into an interval) or a dichotomic output (matched or non-matched). Hereafter, we use the representation of the fingerprint acquired by enrollment as the template $(T)$ and the representation of the input fingerprint $(I)$. Two fingerprints are called genuine if they represent the same finger, and impostor when they are different.

Several factors make fingerprint matching a very challenging problem [113]: image noise, skin condition, distortions, rotations, displacement, etc. There are two well-known properties in fingerprints: large variability in different impressions of the same finger (large intra-class variations) and much similarity between two images from different fingers (small interclass variations).

The most popular and used technique is the minutiae-based matching. Subsequent subsections will detail the main concepts of minutiae-based matching (Subsection 2.1), including the distinction between global and local matching (Subsection 2.2) and feature extraction techniques that are commonly used to obtain the minutiae for matching (Subsection 2.3).

\subsection{Minutiae-based Matching}

The output of a minutiae extraction stage is, at least, a set of minutiae. Each minutia is represented by its location coordinates and orientation angles, forming a 3 -tuple $M=$ $(x, y, \theta) . T$ and $I$ fingerprints have $m$ and $n$ minutiae, respectively. A minutia $M_{j}$ in $I$ is considered matched with a minutia $M_{i}$ in $T$ when it falls within the tolerance box of $M_{i}$. The tolerance box is defined as the maximum spatial distance and direction difference 
permitted to compensate unavoidable errors made by minutiae extractors and positioning changes produced by distortions.

Obviously, it is mandatory to obtain the optimal displacement and rotation alignment of fingerprints in order to maximize the number of minutiae matched. This also includes scaling and advanced geometrical transformations. After alignment, a matching score for the two fingerprints is calculated. To do this, the pairing function between minutiae $M_{i}$ and $M_{j}$ must be found, assuming that each minutia has either exactly one matched minutia in the other fingerprint or has none at all. Achieving the optimal pairing is not a trivial task when the correct alignment is not known, as it usually happens in practice. For instance, a minutia of $I$ may fall within the tolerance box of two or more minutiae of $T$. An assignment algorithm, preferably fast or greedy, is usually employed for this task.

Finally, the matching score could be formulated as follows:

$$
\text { matching_score }=\frac{k}{(n+m) / 2}
$$

where $k$ is the number of matched minutiae. It is a simple expression usually shared among matching algorithms. However, advanced models normally exploit further information such as the minutiae quality and adjusted parameters by using optimization techniques.

\subsection{Global and Local Minutiae Matching}

Fingerprint minutiae matching can be firstly divided into two families of methods:

- Global minutiae matching: the algorithms of this kind tackle the alignment process by taking into consideration all the minutiae as a whole set in a global manner. Since the number of components to be aligned are, at least, three (two directions and the angle), they may require high computational resources and often the usage of a pre-alignment stage that is based on other features extracted such as singular points or orientation maps.

- Local minutiae matching: they consist of comparing two fingerprints according to local structures of minutiae. These structures are formed by considering different relationships based on proximity between closer minutiae. They are characterized by properties that are invariant regarding global transformations, such as translations and rotations. Thus, they do not take into account global relationships and allow to make matching with partial information.

The benefits of local minutiae matching are simplicity, low computational complexity and distortion tolerance, whereas global minutiae matching techniques lead to high distinctiveness. However, all of these benefits could be achieved by using hybrid strategies that perform a local minutiae matching followed by a consolidation stage. The former step determines pairs of minutiae that locally match and extracts a subset of candidate alignments for $I$ and $T$. The latter step, which is not strictly mandatory, is aimed at checking the degree in which local matches support global matching. 
Table 1: Enumeration of representative global minutiae matching algorithms

\begin{tabular}{|c|l|}
\hline References & Main Property \\
\hline$[138,101]$ & Hough transform-based approaches \\
{$[72,107,37]$} & Ridge-based relative pre-alignment \\
{$[47,189]$} & Global matching of clusters of minutiae \\
{$[157,11,28,163]$} & Algebraic geometry-based approaches \\
{$[30,83]$} & Singularity-based relative pre-alignment \\
{$[140,98,118]$} & Warping modeling-based approaches \\
{$[120]$} & Minutiae matching with tesselated local information \\
{$[161]$} & Global minutiae matching with image correlation \\
{$[56,104,175,82]$} & Orientation image-based relative pre-alignment \\
{$[151,145,144]$} & Global matching by evolutionary algorithms \\
{$[78,92]$} & Weighted global matching with adjustment of scores \\
{$[32,160]$} & Hierarchical and/or multilevel minutiae matching \\
\hline
\end{tabular}

Recently, most of the proposals of fingerprint minutiae matching designed to be implemented in real systems have given up the idea of global matching in favor of local matching. Nevertheless, although the focus of this paper is to review the properties and methods belonging to local minutiae matching, we also provide an enumeration of the most influential global minutiae matching methods proposed in the specialized literature (see Table 1).

\subsection{Feature Extraction Techniques}

This section is devoted to briefly identify the subset of feature extraction techniques frequently used in conjunction with fingerprint minutiae matching. It is worth mentioning that an exhaustive review of existing techniques can be found in [113]. Next, we will summarize the most representative algorithms according to their usage in practice and in subsequent matching approaches proposed in the literature:

- Fingerprint segmentation [108, 34].

- Local orientation map estimation $[125,137,4]$.

- Local ridge frequencies estimation [65, 109].

- Singular and core points searching $[85,74,139,86]$.

- Alignment of local orientations and ridge frequencies [27].

- Fingerprint binarization [125, 65].

- Fingerprint skeletonization [180, 58, 106].

- Minutiae extraction [1, 108]. 
- Spurious minutiae removal [153, 12, 184, 95, 129].

\section{Local Minutiae Matching: Properties, Methods and Taxonomy}

In the following, we present the taxonomy of minutiae-based local matching methods and the properties used to build it. First, in Subsection 3.1, the essential characteristics, which will define the categories of the taxonomy, will be outlined. Next, in Subsection 3.2, we will enumerate all the minutiae-based local matching methods proposed in the scientific literature. Then, each method will be categorized according to the studied properties to provide a comprehensive taxonomy.

\subsection{Properties for Categorizing Local Matching}

This subsection provides a framework for the organization of the matching methods that will be presented in Subsection 3.2. The aspects discussed here include (1) topology of local structure, (2) type of consolidation, (3) usage of additional features, (4) minutiae peculiarities and (5) parameter learning. These mentioned facets are involved in the definition of the taxonomy, because they determine the way of operation of each matching technique.

\subsubsection{Topology of local structure}

Local matching is based on the computation of the similarity between local regions of two fingerprints, for the sake of achieving the desired invariance regarding translations and rotations. In minutiae matching, regions are associated with subsets of minutiae that present some kind of relationship, mainly based on location and proximity. Hence, the subsets of minutiae are organized into local structures and they can be built under different assumptions:

- Nearest Neighbors (NN): local structures are formed by a central minutia and a certain number of its nearest neighbor minutiae. The number of neighbors is specified as an input parameter and the local structures are usually defined by distances, directions and angles between pairs of minutiae.

- Fixed Radius: it creates a local structure from a central minutia by using a maximum distance $\left(d_{\max }\right)$ in the graph $\left(V_{i}, E_{i}\right)$ defined as: $(1)$ a set of vertices $V_{i}$ containing all the minutiae whose spatial distance is less than or equal to $d_{\max }$ and, (2) a set of edges $E_{i}$ connecting the central minutia and every vertex in $V_{i}$. The distance $d_{\max }$ is specified as an input parameter and the local structures are defined by the set of edges in clockwise traversing, by using distances as well as absolute and relative angles.

- Texture mixed: a local structure is defined as a feature vector that contains proper information extracted from the minutia and other types of information coming from additional features extracted from the fingerprint image, such as local orientation, ridge frequency, gray-scale image properties or sampling of equidistant points following the ridge starting from the minutia, from neighbor ridges or organized in a circular pattern around a central minutia. This aspect is closely related to the use of additional features 
(third property described in this subsection), which indicates the source of the extra information used in the local structure. Also, if the matcher has the Ridge Properties (within the Peculiarities in Minutia aspect), activated, this is a symptom of using the aforementioned sampling.

- Minutiae Triplets: firstly used for indexing approaches, they are also interesting to yield local structures. Triplets may be built by some type of triangulation or by using all possible combinations of triplets in local regions. The local structures use information regarding angles of the vertices, length of the sides and some triangle properties such as direction, orientation, etc.

- K-Plet: it is an extension of the NN local based structure where it is ensured that the nearest neighbors minutiae are equally distributed in the four quadrants around the minutia.

- Minutia Cylinder: as an extension of fixed radius local structures, it allows a fixed length invariant coding for each minutia based on a discretization of a cuboid into cells. The cylinder is set up by using the radius as the base and the direction difference between minutiae as the height. It also allows binary representation of local structures for fast matching.

\subsubsection{Type of consolidation}

Although the partial scores obtained from the comparison of local structures could straightaway get a final matching score, it is common to develop a further consolidation stage in order to check whether the local similarity is supported at the global level or not. It adds an extra stage to evaluate the coherence among spatial relationships taking the local structures as basic elements. It is very useful in some cases, in which local structures could match in fingerprints from different fingers, independent of the fingerprint region that they represent. Different consolidation techniques have been proposed and can be easily isolated from the rest of the properties studied in this section:

- Single transformation: it is the simplest consolidation idea, based on the alignment of $T$ and $I$ by using the best transformation resulting from a local structure matching. A common procedure is to estimate a very limited number of pairs of local structures that received the highest matching scores and then to use the translation and rotation obtained from them to carry out a global alignment for the remaining minutiae.

- Consensus of transformations: it tries to evaluate to what extent each transformation obtained from a local structure matching is consistent with the others. Another manner is to assess the maximum number of consistent individual transformations. There are different approaches to calculate this estimator, although the most common one is to check that a subset of the most similar local structures remains consistent.

- Multiple transformations: due to the fact that the best transformation coming from the most similar local structures is not the best transformation at the global level, 
multiple transformations may be used by: (1) selecting the final transformation according to the highest score achieved in the final pairing stage, (2) restricting the global matching to regions adjacent to each reference pair, or (3) fusing the results of multiple registrations.

- Complex transformation: this group includes transformations which are based on complex models to alleviate deformations and plastic distortions. For instance, there are models that apply a thin-plate spline to represent elastic deformations, or use the Parzen window to estimate the probability density.

- Incremental consolidation: when arranging the local structures into a graph, connecting the minutiae by the edges, the matching can be performed trough a dual graph traversal algorithm in a breadth-first fashion. At the end of the route, the algorithm returns the number of matched nodes. This process is repeated for every pair of minutiae and the best solution is finally chosen.

\subsubsection{Use of additional features}

We call as additional features those cases in which local structures also incorporate information gathered from other external sources. They may come from other feature extraction processes such as the local orientation image or the local ridge frequency estimation. Once again, we would like to emphasize that the additional features must be external with respect to the minutiae extraction algorithm. Thus, these additional features can cooperate with the mandatory features associated to minutiae (minutiae position and direction) defined by standards like ISO/IEC 19794-2. The external additional features used are the following:

- Ridges Frequency (RF): a local ridges frequency represents the local average pixel distance between ridges. It can be used either as a local feature associated to a certain region (or minutia) of the fingerprint image, when it is relativized with respect to the global ridges frequency of the fingerprint, or to normalize distances between two minutiae as a method of palliating the effect of distortion.

- Core points: the locations and orientations of core singularities are extracted from the fingerprint images for supporting the decision made by the local matching. For instance, they could be used to perform a relative pre-alignment, discarding those minutiae that are far from the original directions, or to involve only those minutiae that are close to them.

- Local Orientation (LO): locally, a fingerprint has a well-defined orientation field given by the ridge direction in a certain region of the image. In order to estimate it, it is normal to define a window size (ranging from $8 \times 8$ to $16 \times 16$ ) in order to quantize the average direction into 8 or 16 angles. The local orientation is then a number associated to a region of the fingerprint and it can be also associated to a central minutia of a local structure. 
- Gray-Scale Images (GSI): they include texture information such as regions of grayscale fingerprint images enhanced by filters, derived from variances among pixels, obtained by Gabor expansion or FingerCode textures [75].

\subsubsection{Peculiarities in minutiae}

Unlike the previous property, we define as a peculiarity in minutiae the additional information closely related to the minutia that can be extracted by using an advanced minutiae extractor. They are considered as supplementary features, different of position and direction, directly obtained from the minutiae set and being essential for the performance of a concrete matching technique. In what follows we present the most important ones:

- Types of minutia: one of the most common peculiarities required by many matchers is the type of minutia, dividing them into two classical types: bifurcations and ridge ends.

- Ridge Count (RC): this peculiarity is associated to each central minutia of the local structure and represents the number of ridges that are cut across the line joining two minutiae. The minutiae extractor requires access to the binarized or skeletonized fingerprint image to be computed.

- Ridge Properties (RP): the ridge which the minutia belongs to is analyzed in terms of its degree of curvature or by sampling some equidistant points along the curve to form relationships with respect to the central minutia. Here, the minutiae extractor requires to explore the skeletonized fingerprint image to walk through the ridges.

\subsubsection{Parameter learning}

Finally, with the term of parameter learning we refer to the application of machine learning based techniques to optimize the separation between genuine and impostor fingerprints. They are usually employed in the optimization of the similarity score that determines the final decision. The parameters typically involved in the learning process are the weights associated to the contribution of each pair of matched minutiae to the computation of the final score. This and other forms of parameter learning are the following:

- Matching Score (MS): a function receiving as input the feature vectors that represent two local structures and obtaining as output the similarity score is learned by means of neural networks or other regression schemes. The learning process is supervised and it is focused on optimizing the final matching score between genuine or impostor fingerprints.

- Local Similarity (LS): an off-line learning process is performed to learn the genuine similarity between local structures or to adjust the contribution weights associated to each component of the feature vector. 


\subsection{Taxonomy of Minutiae-Based Local Matching Methods}

Nowadays, more than 80 minutiae-based local matching methods have been proposed in the specialized literature. This section is focused on enumerating and categorizing them according to the properties studied before. Table 2 presents an enumeration of the methods reviewed in this paper. In this field, the authors do not usually give a name for their proposal, with few exceptions. Thus, we will use the reference of the paper as their identifier.

As we can see in Table 2, the most common proposals use the Texture based topology, being the main baseline method the one proposed in [154]. Regarding other topologies, almost all the NN and Radius approaches provide from the matchers [81] and [136]. Referring to consolidation and the additional features, we can observe that all categories are spread over all methods without a clear norm. The access to the RP is more common in recent methods. Moreover, the RC and the use of the Types of minutiae are in decline in recent years, due to their lack of uniformity in different prints obtained from the same finger. Finally, few techniques require the use of parameter learning.

\section{Related and Current Work on Matching}

Once we have provided a comprehensive review on minutiae-based fingerprint matching methods, it is meaningful to also provide other kinds of procedures using for matching. They can be seen as related techniques that could be connected with matching, and current work in other ways of improving matching in different application areas. In this sense, this section gathers the most relevant developments in different issues (Subsection 4.1), distinguishing among correlation-based matching techniques (Subsection 4.2), indexing algorithms and advanced progresses in matching (Subsection 4.3).

\subsection{Correlation-based Techniques and Matching without Minutiae}

Generically, matching by correlation of images occurs when two fingerprint images are superimposed and their similarity is computed through the correlation between corresponding pixels for different alignments. However, this apparently simple operation rarely leads to acceptable results, mainly due to undesirable changes of global structure and brightness and contrast of the image, both depending on distortions and skin condition. Moreover, this process may involve high computational costs.

In the specialized literature, there are various alternatives coped to palliate some of the problems associated with correlation-based matching. For example, to alleviate the distortion problem, some proposals use local windows around the minutiae [90], singular points alignment before correlation [124] or advanced correlation filters [159]. To reduce the computational complexity, the correlation is performed in local regions in the Fourier domain [168], or using the Fourier-Mellin transform to maintain rotation and translation invariance $[149,84]$, the symmetric phase only filter to reduce noise [66] and the curvelet transform [57]. Recently, there is a promising trend that transforms minutiae positions and orientations to spectral representations in fixed-length feature vectors invariant to translations, rotations and scale. They are suitable to be reduced by dimensionality reduction techniques to speed up the matching process $[171,121]$. 
Table 2: Enumeration and classification of minutiae-based local matching methods

\begin{tabular}{|c|c|c|c|c|c|}
\hline References & $\begin{array}{c}\text { Local Structure } \\
\text { Topology }\end{array}$ & $\begin{array}{c}\text { Type of } \\
\text { Consolidation }\end{array}$ & $\begin{array}{l}\text { Additional } \\
\text { Features }\end{array}$ & $\begin{array}{c}\text { Minutiae } \\
\text { Peculiarities }\end{array}$ & $\begin{array}{c}\text { Parameter } \\
\text { Learning }\end{array}$ \\
\hline$[165]$ & NN & Incremental & None & None & None \\
\hline$[81,6]$ & NN & Single & None & Types + RC & None \\
\hline$[136]$ & Radius & Consensus & None & $\mathrm{RC}$ & None \\
\hline [94] & Texture & Multiple & $\mathrm{RF}$ & Types & None \\
\hline [181] & NN & Single & Core & None & None \\
\hline$[5]$ & NN & Complex & None & None & None \\
\hline$[63]$ & Texture & Single & LO & Types + RP & None \\
\hline [141] & Not defined & Not defined & $\mathrm{RF}+\mathrm{GSI}$ & Not defined & None \\
\hline [150] & Triplets & None & None & $\mathrm{RC}$ & None \\
\hline$[154,166,117]$ & Texture & Multiple & LO & None & None \\
\hline$[29]$ & NN & Single & None & Types + RP & None \\
\hline$[123,182]$ & Texture & Multiple & LO & $\mathrm{RP}$ & None \\
\hline$[127,116]$ & Triplets & Multiple & None & None & None \\
\hline$[142]$ & Texture & Multiple & LO & Types & None \\
\hline [170] & Radius & Multiple & None & None & None \\
\hline [41] & K-Plet & Incremental & Core & Types & None \\
\hline [46] & Triplets & Multiple & None & Types + RC & None \\
\hline$[77]$ & NN & Consensus & None & None & MS \\
\hline$[134,167]$ & Texture & Single & LO & None & None \\
\hline$[132]$ & Texture + Triplets & Single & $\mathrm{LO}$ & None & None \\
\hline$[155,156]$ & Texture & Single & $\mathrm{LO}$ & $\mathrm{RC}$ & None \\
\hline$[178,183]$ & Triplets & Single & None & None & None \\
\hline$[179]$ & NN & Single & None & Types & None \\
\hline$[13]$ & NN & None & None & $\mathrm{RC}$ & None \\
\hline$[35,15]$ & Radius & Consensus & None & None & None \\
\hline$[36]$ & Texture + Triplets & Consensus & LO & None & LS \\
\hline$[39]$ & K-Plet & Incremental & None & Types & None \\
\hline [40] & Texture & Single & GSI & None & None \\
\hline$[50,133,187]$ & Texture & Multiple & None & $\mathrm{RP}$ & None \\
\hline$[62,96]$ & Texture & Consensus & GSI & $\mathrm{RP}$ & None \\
\hline$[93]$ & K-Plet & Complex & None & None & None \\
\hline [143] & NN & Consensus & None & $\mathrm{RC}$ & None \\
\hline$[7]$ & Texture & Multiple & GSI & None & None \\
\hline$[48,2]$ & Texture & None & None & $\mathrm{RP}$ & None \\
\hline$[61]$ & Texture & Complex & GSI & $\mathrm{RP}$ & None \\
\hline [131] & Radius & Consensus & LO & None & None \\
\hline$[135]$ & Texture & Multiple & None & Types + RP & None \\
\hline$[172,177]$ & Triplets & Incremental & None & None & None \\
\hline$[164]$ & Texture & Consensus & LO & $\mathrm{RP}$ & None \\
\hline$[8]$ & Texture & Single & Core + GSI & $\mathrm{RC}$ & None \\
\hline [49] & Radius + Texture & Multiple & $\mathrm{RF}+$ Core + LO & Types + RP & MS \\
\hline [88] & $\begin{array}{l}\text { Triplets } \\
\end{array}$ & None & None & Types & None \\
\hline$[89]$ & Radius & Incremental & None & None & None \\
\hline [115] & K-Plet & Single & GSI & Types & None \\
\hline [162] & K-Plet & Single & $\mathrm{RF}+\mathrm{GSI}$ & $\mathrm{RP}$ & None \\
\hline [188] & NN & None & None & Types $+\mathrm{RC}$ & None \\
\hline$[14]$ & Texture & Consensus & None & $\mathrm{RC}+\mathrm{RP}$ & None \\
\hline$[20,152]$ & Texture & Complex & None & $\mathrm{RP}$ & None \\
\hline$[21]$ & Radius + Texture & Multiple & $\mathrm{RF}+\mathrm{LO}$ & $\mathrm{RP}$ & MS \\
\hline$[87]$ & NN & Single & GSI & Types + RC & None \\
\hline [114] & Texture & Incremental & None & Types + RP & None \\
\hline [146] & Radius & None & None & None & None \\
\hline$[147]$ & Texture & None & LO & None & None \\
\hline$[173]$ & Radius & Consensus & None & Types + RC & None \\
\hline [186] & Triplets & Multiple & None & Types & None \\
\hline$[19]$ & Texture & Multiple & $\mathrm{RF}+\mathrm{LO}$ & $\mathrm{RP}$ & None \\
\hline$[26,67]$ & Cylinder & Consensus & None & None & None \\
\hline$[148]$ & NN & Multiple & None & None & None \\
\hline$[31]$ & Texture + Triplets & None & GSI & None & None \\
\hline$[42]$ & K-Plet & Incremental & None & $\mathrm{RC}+\mathrm{RP}$ & None \\
\hline [53] & NN & None & None & None & None \\
\hline [100] & Texture + Radius & Consensus & GSI & None & None \\
\hline [185] & Triplets & Single & $\mathrm{LO}$ & None & None \\
\hline$[17]$ & Texture & Multiple & $\mathrm{LO}+$ Core & $\mathrm{RP}$ & None \\
\hline [18] & Radius + Texture & Incremental & Core + LO & $\mathrm{RP}$ & None \\
\hline$[23]$ & Texture + Cylinder & Consensus & $\mathrm{RF}+\mathrm{LO}+$ Core & None & None \\
\hline [33] & Texture & None & GSI & None & None \\
\hline [43] & Radius & Multiple & None & Types & None \\
\hline [55] & Radius + Texture & None & LO + GSI & None & None \\
\hline [119] & Texture + Triplets & None & Core + GSI & None & None \\
\hline
\end{tabular}


Other approaches perform fingerprint matching without the use of minutiae. They use the so-called texture information, being the most popular the FingerCode approach [75], which chains tessellated areas related to core points with Gabor filter to capture useful texture information. FingerCode features have been used in later research [7, 122, 176]. Isolated orientation [91] or ridge information [169] can also be used for matching. Finally, when high resolution images are available, level-3 features such as sweat pores, dots and incipient ridges can be used instead of minutiae $[68,103]$.

\subsection{Fingerprint Indexing}

Fingerprint indexing arises from the necessity of quick access to the fingerprint templates database in identification tasks. Some indexing techniques use partial information provided by the extracted minutiae of the fingerprint and build local structures centered on each minutia to establish similarity relationships between fingerprints and key indexes. This allows the ordering of candidate templates to increase the probability to match true paired fingerprints. Actually, these approaches can be viewed as minutiae-based matching approaches if the matching score is proportionally related to the number of coincident local structures.

The pure indexing proposals found in the literature are those based on minutiae triplets, which consider triangle-based characteristics to compute similarity among fingerprints, such as lengths, angles, handleless [10], etc.; and triangulations to improve efficiency [99]. Other indexing approaches utilize LO [105] and also RF [22]. Finally, several criteria for narrowing the candidate list obtained from indexing are evaluated in [24].

\subsection{Current Progress in Matching}

Nowadays, the matching field is continually in progress, offering new developments to improve personal identification. In the following, we briefly mention different matching related issues being currently tackled:

- Accelerating fingerprint matching: many efforts have been performed to speed up the matching process, for instance, by means of FPGA-based [79], GPU-based [59] parallel architectures or distributed computing [130].

- Fingerprint matching in embedded systems: sensors [3] and smart cards [9].

- Latent fingerprint matching: it is a more complicated problem because these fingerprints are inadvertent impressions left by fingers on surfaces [70, 128].

- Palmprint matching: based on ridges [44], minutiae [25, 32] and also effective approaches for latent matching [69, 102].

- Combinations with other traits and multiple matching: with face recognition [64], multiple matching [73], multiple sample [38] and minutiae-based synthesis for matching [158]. 
- Privacy protection in fingerprint matching: which tries to avoid the traditional encryption with its associated decryption, which exposes the fingerprint to the attacker. Two examples of recent techniques are the reverse MCC representation [51] and the combination of two different fingerprints into a new identity, based on minutiae, orientations and singular points [97].

\section{Experimental Evaluation of Local Minutiae Matching Methods}

This section is devoted to perform an experimental evaluation of the most important local minutiae-based matching algorithms. Subsection 5.1 establishes the experimental framework, presenting information about the used databases, the performance measures, the algorithms and their parameters. Then, Subsection 5.2 shows the analysis of the results of the used methods over the public FVC databases. Subsection 5.3 presents a study over four databases captured by the authors.

\subsection{Experimental Set Up}

This section describes the databases (Subsection 5.1.1), the accuracy measures (Subsection 5.1.2) and the framework (Subsection 5.1.3) used to carry out the experimental evaluation of the matchers.

\subsubsection{Databases}

We have used a wide variety of databases to test the performance and behavior of the matching algorithms. Table 3 presents their characteristics, showing their size and the average number of minutiae of the template and input fingerprints.

Table 3: Summary description of the used databases.

\begin{tabular}{|l|r|r|r|r|}
\hline Denomination & $\begin{array}{r}\text { Number of } \\
\text { Fingerprints }\end{array}$ & $\begin{array}{r}\text { Impressions } \\
\text { per finger }\end{array}$ & $\begin{array}{r}\text { Average template } \\
\text { minutiae number }\end{array}$ & $\begin{array}{r}\text { Average input } \\
\text { minutiae number }\end{array}$ \\
\hline FVC2000_db1a & 8 & 49.51 & 48.93 \\
FVC2000_db2a & 100 & 8 & 58.43 & 57.97 \\
FVC2000_db3a & 100 & 8 & 132.97 & 144.18 \\
FVC2000_db4a & 100 & 8 & 36.88 & 37.10 \\
FVC2002_db1a & 100 & 8 & 53.11 & 49.69 \\
FVC2002_db2a & 100 & 8 & 61.87 & 56.93 \\
FVC2002_db3a & 100 & 8 & 58.23 & 57.52 \\
FVC2002_db4a & 100 & 8 & 50.52 & 49.78 \\
FVC2004_db1a & 100 & 8 & 49.01 & 62.84 \\
FVC2004_db2a & 100 & 8 & 64.45 & 64.19 \\
FVC2004_db3a & 100 & 8 & 94.52 & 98.63 \\
FVC2004_db4a & 100 & 10 & 55.00 & 52.61 \\
\hline \hline DB1 & 100 & 10 & 45.26 & 45.20 \\
DB2 & 1228 & 10 & 145.79 & 142.94 \\
DB3 & 1228 & 10 & 44.36 & 43.34 \\
DB4 & 1228 & 44.50 & 43.35 \\
\hline
\end{tabular}

First, we apply the algorithms over twelve of the well-known FVC databases, using the first impression of each finger as template, and the other seven impressions as input. These 
databases are designed for verification competitions, and therefore their fingerprints have bad quality on purpose. More information about the FVCs databases can be found in $[110,112,111]$

Four additional databases, captured by the authors' research groups, are used for the study. They simulate a real environment for identification with consented fingerprints captures of reasonable quality. All of them are composed by the same fingers, captured by four different sensors (Table 4).

Table 4: Sensors used to capture the fingerprints.

\begin{tabular}{|l|l|l|l|}
\hline Database & Sensor & Sensor type & Fingerprint type \\
\hline DB1 & Upek Eikon & Capacitive & Swipe \\
DB2 & Suprema RealScan-D & Optical & Rolled \\
DB3 & Suprema BioMini & Optical & Plain \\
DB4 & SecuGen Hamster IV & Optical & Plain \\
\hline
\end{tabular}

A total of 308 people participated in the study. The fingerprints of the thumb, forefinger and middle finger of both their hands were captured along three different sessions. After removing the failed captures, we selected three random input fingerprints per session and a single template fingerprint for each finger and sensor. After this manner we get four final databases that contain the same 1228 fingers captured by four different sensors.

\subsubsection{Accuracy measures}

The accuracy of a fingerprint matcher can be measured from two different perspectives:

- Verification: consists of matching two fingerprints to determine whether they correspond to the same finger or not.

- Identification: tries to find the match of an input fingerprint in a database, comparing it to all the templates.

Each perspective employs different accuracy measures. In this paper, we use the following verification measures:

- False Matching Rate (FMR): rate of different fingerprints that are considered to be the same by the matcher. Each possible score has an FMR associated; the higher the score, the lower the FMR.

- False Non-Matching Rate (FNMR): rate of corresponding fingerprints that are erroneously considered different.

- Equal-Error Rate (EER): value (corresponding to a certain score threshold) where FMR and FNMR are equal.

- ROC: curve that plots the Genuine Matching Rate (GMR $=1-$ FNMR) versus the FMR. 
- FMR100: lowest achievable FNMR for a FMR $\leq 1 \%$.

- FMR1000: lowest achievable FNMR for a FMR $\leq 0.1 \%$.

- ZeroFMR: lowest achievable FNMR for a FMR $=0 \%$.

Within an identification process, most of the accuracy measures are related to the rank, which is the position of the genuine score if all the obtained scores are ordered in descending order. In other words, the rank is the minimum number of database fingerprints that have to be returned by the identification system to ensure that the correct identity is included. We use the following identification accuracy measures:

- True positive rate (TPR): percentage of test fingerprints that are correctly identified in the database, when only the best matching score is retrieved. The TPR is the error obtained when using a rank of 1 .

- R100: lowest rank that allows an error lower than $1 \%$.

- ZeroR: lowest rank that does not allow errors.

- Cumulative Match Curve (CMC): curve that represents the error associated to each rank.

The optimum value for R100 and ZeroR is 1 , whereas the worst one is the size of the database.

In addition to all these values, the average matching time is also important to determine if a matching algorithm is suitable for a certain identification system.

For reasons of space and concision, not all of these measures are presented in the paper. The full set of results is accessible at http://sci2s.ugr.es/MatchingReview/.

Statistical tests allow to establish a fair comparison between the methods and to detect significant differences. In this paper, we use the nonparametric tests recommended in $[45,54]$, which claim to be simple, safe and robust.

Furthermore, we apply the Friedman test [52] to measure the differences between the methods with a multiple comparison analysis. The Holm procedure is applied to find out which algorithms are distinctive. ${ }^{1}$

\subsubsection{Experimental framework}

To compute these measures it is necessary to perform all the matching comparisons between template and input fingerprints. In order to obtain the results within a reasonable time, and to fix a common execution environment, all the experiments have been carried out within the parallel framework proposed in [130], which speeds up the computation while ensuring that the results are the same as in a sequential execution. The NIGOS

\footnotetext{
${ }^{1}$ Additional information about these tests, as well as the corresponding software, are available at http: //sci2s.ugr.es/sicidm/.
} 
mindtct algorithm [165] has been used for the minutiae extraction. All executions have been performed in a cluster of 12 machines, each of them with two Intel(R) Xeon(R) E5-2620 $\mathrm{CPU}$ at $2.00 \mathrm{GHz}$ and 64GB RAM.

The empirical study involves 12 matching algorithms from those listed in Table 2. We want to outline that all the implementations of the matching algorithms, excepting the proposed in [165], were developed by us and they are only based on the descriptions and specifications given by the respective authors according to their papers. It is also noteworthy that our implementation of Feng's algorithm only uses the minutiae features provided by the minutiae extractor, and therefore is not as complex as the original algorithm. The parameter values used for all matchers have been extracted from these papers and are shown in Table 5. In the cases where the parameter values are not given in the original paper, we experimentally selected values that suit the general case. We have not performed any training to adapt these parameters, because our objective is not to maximize the accuracy, but to fairly compare the matchers and their robustness in a common environment and upon different databases.

\subsection{Analysis and Empirical Results on FVC Databases}

This section analyzes the results obtained over the 12 FVC databases, in terms of verification and identification.

\subsubsection{Verification}

Tables 6 and 7 present the EER and FMR100, respectively, as the error percentage obtained for all tested algorithms over the 700 input fingerprints of each FVC database. The best result for each database is stressed in boldface. Additionally, Figure 1 plots the ROC curve for the most difficult FVC database (FVC2002_db3a, which obtains the highest average EER).
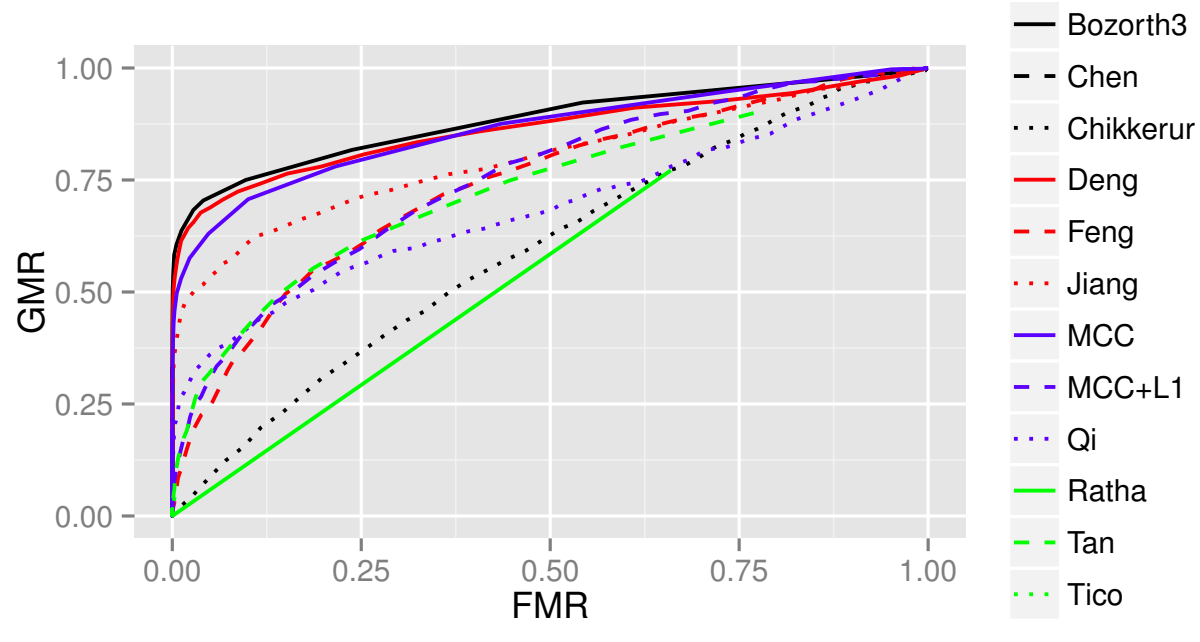

Figure 1: ROC curve for FVC2002_db3a

Bozorth3 is the best performing algorithm in general. If we focus on the EER, MCC also obtains good results, while Deng is more accurate in terms of FMR100. The ROC curves 
Table 5: Parameters for the methods used in the experiments

\begin{tabular}{|c|c|}
\hline Algorithm & Parameters \\
\hline Mindtct $[165]$ & $\begin{array}{l}\text { output format }=\text { ANSI INCITS } 378-2004 \\
\text { image enhancement }=\text { enabled }\end{array}$ \\
\hline Bozorth3 [165] & $\begin{array}{l}\text { input format }=\text { ANSI INCITS 378-2004, Maximum number of minutiae }=150 \text {, } \\
\text { Minimum number of minutiae }=10\end{array}$ \\
\hline Jiang $[81]$ & $\begin{array}{l}w_{d}=1, w_{\theta}=54 \pi, w_{\phi}=54 \pi, w_{n}=0, w_{t}=0 \\
\text { Consolidation step iterations }=5, \text { Minutiae neighborhood size }=2 \\
B G_{1}=8, B G_{2}=\frac{\pi}{6}, B G_{3}=\frac{\pi}{6}\end{array}$ \\
\hline Ratha $[136]$ & $\begin{array}{l}\text { Neigh }=6, F_{\min }=0.4, \mathrm{TM}=8, \text { RelDist }=0.2, \text { RidgesDiff }=10 \\
\text { EdgesDiff }=0.1, \text { MisMatch }=10000\end{array}$ \\
\hline Tan $[150]$ & $\begin{array}{l}\Delta_{\alpha}=10, \Delta_{\tau}=20, \text { WindowsSize }=32, \text { Triplet }_{\text {angle }}=2, \\
\text { Triplet }_{\text {side }}=15, \text { Triplet }_{\text {minutieaDensity }}=2, \text { Triplet }_{\text {RidgeCount }}=2, \\
\mathrm{TS}=0.15, T_{\Theta}=30, T_{1}=150, T_{2}=100, T_{D}=12, T_{N}=8, \text { MaxTriangleWidth }=300\end{array}$ \\
\hline Tico $[154]$ & $\begin{array}{l}T H_{R V}=25, \text { Block }=16, \text { NumRadius }=4, T H R_{t}=\Pi, T H R_{\text {Dist }}=6 \\
T H R_{\text {angle }}=\frac{\Pi}{6}, M T I=6, \mu=0.25\end{array}$ \\
\hline Deng $[46]$ & $\begin{array}{l}\text { Minutiae }_{\text {minDelanuy }}=20, T H_{1}=36, T H_{\text {num }}=20, T H_{\text {edge }}=15 \\
W_{0}=1, W_{1}=W_{2}=\frac{0.3 \cdot 180}{\Pi}, W_{3}=3, W_{4}=W_{5}=6 \\
T H_{d}=8, T H_{\Theta}=T H_{\phi}=T H_{\text {ang }}=\frac{\Pi}{6}, T H_{r c}=3, T H_{S L}=0.2\end{array}$ \\
\hline Qi $[132]$ & $\begin{array}{l}T H_{R V}=25, \text { Block }=16, \text { PointSeg=3,MinutiaeSeg }=6, \text { LongSeg }=18 \\
T H R_{t}=\Pi, T H R_{O}=\frac{\Pi}{2}, T H R_{\text {Dist }}=10, T H R_{\text {angle }}=\frac{\Pi}{4} \\
W_{M}=0.6, W_{O}=0.4\end{array}$ \\
\hline Chen $[35]$ & $\begin{array}{l}T h r_{L}=55, T h r_{H}=80, R=80, R S=100, \theta_{L}=0.25, \theta_{H}=0.4 \\
\operatorname{len}_{L}=5, \operatorname{len}_{H}=20, T h r_{\text {topo }}=0.7\end{array}$ \\
\hline Chikkerur [39] & $K=8$, Bounding box $=\{8, \pi / 6, \pi / 6\}$ \\
\hline Feng $[49]$ & $\begin{array}{l}\text { Neighborhood radius }=60, \text { Translation Tolerance Box }=8 \\
\text { Rotation Tolerance Box }=\pi / 6, \text { Rotation maximum threshold }=5 \pi / 9 \\
\text { Minimum normalized similarity }=\pi\end{array}$ \\
\hline MCC $[26]$ & $\begin{array}{l}R=70, N_{s}=16, N_{d}=6, \sigma_{s}=\frac{28}{3}, \sigma_{d}=\frac{2 \pi}{9}, \mu_{\Psi}=0.01, \tau_{\Psi}=400 \\
\omega=50, \min _{V C}=0.75, \min _{M}=2, \min _{M E}=0.60, \sigma_{\theta}=\frac{\pi}{2}, \max _{n_{p}}=12 \\
\text { Floating-point-based version: enabled, consolidation scheme= LSSR, } \mu_{P}=20 \\
w_{R}=0.5, \mu_{1}^{\rho}=5, \tau_{P}=0.6, \min _{n_{p}}=4 \\
\mu_{2}^{\rho}=\frac{\pi}{12}, \mu_{3}^{\rho}=\frac{\pi}{12}, \tau_{1}^{\rho}=-1.6, \tau_{2}^{\rho}=-30, \tau_{3}^{\rho}=-30, n_{\text {rel }}=5\end{array}$ \\
\hline $\mathbf{M C C}+\mathbf{L} \mathbf{1}[23]$ & $\begin{array}{l}\text { Block }_{w}=16, \text { Block }_{l}=32, F V_{\text {long }}=36, F V_{\text {radius }}=4 \\
W_{1}=W_{3}=0.16, W_{2}=0.37, W_{4}=0.31, \text { Threshold }=0.4\end{array}$ \\
\hline
\end{tabular}

show that Bozorth3, MCC and Deng dominate all methods, followed by Jiang.

These four algorithms are substantially different from each other. For example, MCC uses cylinders as local structure, while Deng uses the texture and Jiang and Bozorth3 use 
Table 6: EER percentages for FVC databases

\begin{tabular}{|c|c|c|c|c|c|c|c|c|c|c|c|c|}
\hline Database & Bozorth3 & Jiang & Ratha & Tan & Tico & Deng & Qi & Chen & Chikkerur & Feng & MCC & $\mathrm{MCC}+\mathrm{L} 1$ \\
\hline FVC2000_db1a & 7.481 & 12.945 & 43.557 & 25.711 & 83.286 & 7.633 & 25.446 & 69.143 & 37.840 & 20.013 & 8.207 & 24.804 \\
\hline FVC2000_db2a & 8.751 & 16.451 & 42.499 & 37.143 & 87.429 & 9.308 & 20.124 & 66.143 & 39.406 & 22.252 & 8.578 & 20.725 \\
\hline FVC2000_db3a & 18.750 & 24.954 & 41.996 & 30.965 & 95.857 & 14.814 & 29.978 & 64.714 & 43.219 & 40.022 & 20.216 & 23.152 \\
\hline FVC2000_db4a & 5.817 & 8.166 & 42.042 & 24.228 & 91.857 & 17.006 & 41.777 & 46.571 & 36.498 & 32.259 & 6.026 & 20.144 \\
\hline FVC2002_db1a & 15.286 & 16.312 & 41.761 & 26.366 & 80.000 & 16.676 & 34.640 & 63.571 & 40.776 & 15.067 & 15.325 & 23.287 \\
\hline FVC2002_db2a & 14.564 & 13.404 & 38.141 & 27.708 & 79.571 & 12.959 & 27.852 & 46.000 & 37.840 & 15.254 & 12.553 & 22.166 \\
\hline FVC2002_db3a & 20.062 & 27.686 & 46.093 & 33.002 & 95.286 & 21.258 & 37.346 & 86.714 & 43.462 & 31.922 & 21.867 & 32.015 \\
\hline FVC2002_db4a & 21.003 & 23.281 & 42.641 & 29.839 & 88.857 & 24.352 & 39.921 & 90.286 & 36.369 & 23.692 & 23.988 & 26.181 \\
\hline FVC2004_db1a & 17.374 & 24.999 & 44.405 & 40.286 & 98.429 & 20.409 & 42.900 & & 47.938 & 23.209 & 19.562 & 28.592 \\
\hline FVC2004_db2a & 17.183 & 23.798 & 45.195 & 38.728 & 48.000 & 20.766 & 35.354 & 85.143 & 42.102 & 29.003 & 19.786 & 31.675 \\
\hline FVC2004_db3a & 6.265 & 13.834 & 43.545 & 31.792 & 79.000 & 9.396 & 31.119 & 29.714 & 43.415 & 35.287 & 10.037 & 18.699 \\
\hline FVC2004_db4a & 26.189 & 31.438 & 42.315 & 33.712 & 65.286 & 28.372 & 40.106 & 93.286 & 40.029 & 29.240 & 28.122 & 27.160 \\
\hline
\end{tabular}

Table 7: FMR100 percentages for FVC databases

\begin{tabular}{|c|c|c|c|c|c|c|c|c|c|c|c|c|}
\hline Database & Bozorth3 & Jiang & Ratha & Tan & Tico & Deng & Qi & Chen & Chikkerur & Feng & MCC & $\mathrm{MCC}+\mathrm{L} 1$ \\
\hline FVC2000_db1a & 13.334 & 20.269 & 98.704 & 67.550 & 90.591 & 12.764 & 50.339 & 100.000 & 97.107 & 76.583 & 14.239 & 79.610 \\
\hline FVC2000_db2a & 18.712 & 27.582 & 98.647 & 80.952 & 94.609 & 18.435 & 47.920 & 100.000 & 96.458 & 89.613 & 18.564 & 71.068 \\
\hline FVC2000_db3a & 37.876 & 54.204 & 98.619 & 89.996 & 98.250 & 28.632 & 95.592 & 100.000 & 98.114 & 97.057 & 46.071 & 82.449 \\
\hline FVC2000_db4a & 14.012 & 15.944 & 98.621 & 77.234 & 100.000 & 31.254 & 88.820 & 100.000 & 95.597 & 89.744 & 9.657 & 66.374 \\
\hline FVC2002_db1a & 24.967 & 25.806 & 98.605 & 67.114 & 92.114 & 22.380 & 56.529 & 100.000 & 97.442 & 52.155 & 22.192 & 76.952 \\
\hline FVC2002_db2a & 22.645 & 20.300 & 98.378 & 64.810 & 94.427 & 17.357 & 46.073 & 56.559 & 97.010 & 48.794 & 19.363 & 72.116 \\
\hline FVC2002_db3a & 37.324 & 56.179 & 98.830 & 85.811 & 100.000 & 39.946 & 74.281 & 100.000 & 97.974 & 90.282 & 47.915 & 86.162 \\
\hline FVC2002_db4a & 52.152 & 51.010 & 98.655 & 80.990 & 96.435 & 51.858 & 75.166 & 100.000 & 96.911 & 85.916 & 51.861 & 86.607 \\
\hline FVC2004_db1a & 36.286 & 51.935 & 98.748 & 92.937 & 98.631 & 41.550 & 77.401 & 100.000 & 98.048 & 87.091 & 36.503 & 86.848 \\
\hline FVC2004_db2a & 35.089 & 52.450 & 98.787 & 91.740 & 97.416 & 37.457 & 74.450 & 91.571 & 97.785 & 95.038 & 41.324 & 86.201 \\
\hline FVC2004_db3a & 13.618 & 34.910 & 98.704 & 86.950 & 95.782 & 28.127 & 79.643 & 40.986 & 98.521 & 98.007 & 28.420 & 71.332 \\
\hline FVC2004_db4a & 60.790 & 66.630 & 98.637 & 85.705 & 97.055 & 69.738 & 83.779 & 100.000 & 97.156 & 87.176 & 66.055 & 89.465 \\
\hline
\end{tabular}

the nearest neighbors. The consolidation type is also different. However, it is noteworthy that none of them use any additional features: Jiang and Deng use both the minutia type and the ridge count, while Bozorth3 and MCC only use the basic minutia information.

It is also interesting that, even though $\mathrm{MCC}+\mathrm{L} 1$ obtains good results when the GMR is high, it does not improve the results obtained with the bare use of MCC. Note that the $\mathrm{MCC}+\mathrm{L} 1$ algorithm uses a different, less accurate variant of MCC (with binary encoding and a different consolidation), meant to be very efficiently implemented on hardware.

This states that none of the characteristics described in Subsection 3.1 can be discarded as worse than the rest: the verification performance is determined by the matching algorithm as a whole, and each local structure and consolidation can supply useful information. Nevertheless, the use of additional features does not always lead to more accurate results.

Along with the accuracy, the computational performance is a very important characteristic of a fingerprint matching algorithm, especially when it has to deal with large fingerprint 
databases.

Table 8 summarizes the average matching times for the tests performed so far. Note that these times are measured in computational time, and therefore are not affected by the parallel framework in which the tests have been carried out.

Table 8: Average matching times (in milliseconds) for FVC databases

\begin{tabular}{|l|rrrrrrrrrrrr|}
\hline Database & Bozorth3 Jiang & Ratha & Tan & Tico & Deng & Qi & Chen & Chikkerur & Feng MCC MCC+L1 \\
\hline FVC2000_db1a & 1.026 & $\mathbf{0 . 3 8 2}$ & 2.865 & 161.339 & 21.319 & 2.409 & 0.739 & 3.936 & 5.051 & 3.577 & 10.094 & 0.762 \\
FVC2000_db2a & 1.719 & $\mathbf{0 . 5 4 1}$ & 3.938 & 513.535 & 34.927 & 4.400 & 0.913 & 7.712 & 6.187 & 6.632 & 13.710 & 0.979 \\
FVC2000_db3a & 6.187 & $\mathbf{4 . 1 4 9}$ & 22.220 & 81277.765 & 228.140 & 91.468 & 4.653 & 51.330 & 91.320 & 46.758 & 82.510 & 4.854 \\
FVC2000_db4a & 3.145 & $\mathbf{0 . 2 3 4}$ & 1.606 & 49.119 & 11.242 & 1.284 & 0.466 & 2.249 & 3.330 & 2.409 & 5.733 & 0.436 \\
\hline FVC2002_db1a & 1.349 & $\mathbf{0 . 4 2 2}$ & 3.189 & 279.543 & 21.319 & 3.386 & 0.749 & 4.541 & 5.532 & 4.047 & 10.880 & 0.784 \\
FVC2002_db2a & 1.233 & $\mathbf{0 . 5 5 1}$ & 4.149 & 442.721 & 33.742 & 3.989 & 0.955 & 4.777 & 6.725 & 4.554 & 14.559 & 0.901 \\
FVC2002_db3a & 1.235 & $\mathbf{0 . 5 3 4}$ & 3.964 & 436.841 & 28.712 & 4.222 & 0.986 & 6.771 & 6.301 & 5.920 & 14.168 & 1.054 \\
FVC2002_db4a & 1.268 & $\mathbf{0 . 3 9 7}$ & 2.915 & 338.823 & 18.921 & 3.697 & 0.784 & 5.163 & 5.046 & 4.770 & 10.334 & 0.745 \\
\hline FVC2004_db1a & 1.488 & $\mathbf{0 . 4 9 1}$ & 3.656 & 313.619 & 16.544 & 3.849 & 0.942 & 6.434 & 5.563 & 5.553 & 12.253 & 0.801 \\
FVC2004_db2a & 1.534 & $\mathbf{0 . 6 8 0}$ & 4.845 & 853.888 & 33.527 & 5.448 & 1.292 & 9.432 & 7.482 & 7.922 & 17.974 & 1.275 \\
FVC2004_db3a & 16.566 & $\mathbf{1 . 8 5 0}$ & 10.815 & 10575.240 & 99.901 & 21.829 & 2.336 & 27.278 & 18.136 & 25.183 & 40.523 & 2.535 \\
FVC2004_db4a & 1.312 & $\mathbf{0 . 4 6 1}$ & 3.340 & 540.880 & 25.047 & 4.189 & 0.872 & 6.484 & 5.325 & 5.913 & 11.737 & 0.840 \\
\hline
\end{tabular}

We can notice that in all cases, Jiang is the fastest algorithm, followed by Qi. The former performs a simple consolidation and does not use any additional features, which makes the computation very fast. The latter does not involve any consolidation, and therefore performs all the matching process from a local point of view.

In the other extreme, the Tan's algorithm is extremely slow, especially for databases with more minutiae per fingerprint. This algorithm computes all the triplets of the fingerprints, and compares them. This computation has factorial order and therefore takes a long time for fingerprints with a certain number of minutiae. This is an example of an algorithm that could be improved by a previous minutiae filtering.

It is curious to note that the Qi's algorithm is very fast, although it also uses triplets. However, it includes a first candidate selection using the texture, avoiding the creation of all possible triplets.

If we compare the overall performance of the algorithms, we can observe that the consolidation bears a high weight in the runtime. Complex consolidations require more computing time, as for MCC, Deng and Tico.

Another observation that can be made is that MCC+L1 is considerably faster than MCC. This is due to the structure of MCC+L1, which first compares the L1 features of the fingerprints, and applies MCC only if they are similar enough. This hierarchical matching is able to save a lot of computing time, but also explains why MCC $+\mathrm{L} 1$ is often less accurate than MCC.

Table 9 shows the results of the statistical tests for several accuracy measures, highlighting Bozorth3, MCC and Deng as the best algorithms. 
Table 9: Statistical tests over the verification measures over the FVC databases

\begin{tabular}{|l|r|r|r|r|}
\hline Algorithm & EER & FMR100 & FMR1000 & ZeroFMR \\
\hline Bozorth3 & $\mathbf{1 . 5 0 0}$ & $\mathbf{2 . 0 8 3}$ & $\mathbf{1 . 7 5 0}$ & $\mathbf{1 . 2 9 2}$ \\
Jiang & 4.000 & 3.500 & 3.833 & 3.458 \\
Ratha & 9.917 & 11.083 & 11.250 & 10.042 \\
Tan & 7.250 & 6.833 & 7.250 & 7.167 \\
Tico & 11.750 & 9.750 & 10.208 & 10.042 \\
Deng & 3.000 & $\mathbf{2 . 0 8 3}$ & 2.250 & 3.083 \\
Qi & 7.583 & 5.583 & 6.333 & 8.833 \\
Chen & 10.833 & 10.500 & 7.250 & 5.500 \\
Chikkerur & 9.000 & 9.917 & 10.625 & 10.042 \\
Feng & 5.333 & 7.500 & 7.833 & 9.458 \\
MCC & 2.500 & 2.333 & 2.167 & 2.250 \\
MCC+L1 & 5.333 & 6.833 & 7.250 & 6.833 \\
\hline Friedman P-value & $6.18 \mathrm{e}-011$ & $6.13 \mathrm{e}-11$ & $5.33 \mathrm{e}-11$ & $7.34 \mathrm{e}-11$ \\
\hline
\end{tabular}

\subsubsection{Identification}

Tables 10 and 11 summarize the R100 and TPR values, respectively. Finally, Figure 2 displays the CMC curves for the FVC2002_db3a database.

Table 10: R100 values for FVC databases (100 templates)

\begin{tabular}{|l|rrrrrrrrrrrrr}
\hline Database & Bozorth3 Jiang & Ratha & Tan & Tico & Deng & Qi & Chen & Chikkerur & Feng & MCC & MCC+L1 \\
\hline FVC2000_db1a & 100 & 91 & 100 & 100 & 96 & 100 & 100 & 100 & 100 & 100 & $\mathbf{7 2}$ & 79 \\
FVC2000_db2a & 100 & 96 & 100 & 100 & 94 & 72 & 97 & 100 & 100 & 100 & $\mathbf{6 4}$ & 77 \\
FVC2000_db3a & 100 & 98 & 100 & 94 & 97 & $\mathbf{8 1}$ & 98 & 100 & 100 & 100 & 98 & 84 \\
FVC2000_db4a & 100 & 79 & 100 & 100 & 100 & 100 & 100 & 100 & 100 & 100 & $\mathbf{3 3}$ & 95 \\
\hline FVC2002_db1a & 100 & 95 & 100 & 100 & 100 & 100 & 100 & 100 & 100 & 100 & 89 & $\mathbf{8 5}$ \\
FVC2002_db2a & 100 & 92 & 100 & 100 & 100 & 100 & 98 & 100 & 100 & 100 & 88 & $\mathbf{8 0}$ \\
FVC2002_db3a & 100 & 98 & 100 & 100 & 99 & 100 & 99 & 100 & 100 & 100 & 91 & $\mathbf{8 6}$ \\
FVC2002_db4a & 100 & 95 & 100 & 100 & 98 & 100 & 100 & 100 & 100 & 100 & 91 & $\mathbf{8 4}$ \\
\hline FVC2004_db1a & 100 & 94 & 100 & 100 & 100 & 100 & 99 & 100 & 100 & 100 & $\mathbf{8 7}$ & 91 \\
FVC2004_db2a & 100 & 96 & 100 & 100 & 99 & 98 & 99 & 100 & 100 & 100 & $\mathbf{9 4}$ & $\mathbf{9 4}$ \\
FVC2004_db3a & 100 & 90 & 100 & 100 & 96 & $\mathbf{4 9}$ & 99 & 100 & 100 & 100 & 64 & 84 \\
FVC2004_db4a & 100 & 96 & 100 & 100 & 96 & 100 & 99 & 100 & 100 & 100 & 94 & $\mathbf{8 9}$ \\
\hline
\end{tabular}

It is curious to observe that, while $\mathrm{MCC}+\mathrm{L} 1$ is the best algorithm if we focus on the rank, MCC obtains better numeric results (for example for FVC2000_db4a) and Deng and Bozorth3 have higher TPR in most cases. The CMC curves explain this behavior. For low ranks, Deng and Bozorth3 perform better, and therefore have a lower TPR. MCC is slightly below Deng in accuracy, while MCC+L1 obtains good results for very high ranks.

The high values obtained denote the difficulty of the FVC databases: the algorithms need to return the majority of the databases in order to ensure that the genuine fingerprint is returned. Note that the methods that have a value of 100 return the entire database. Chen's algorithm has a very low CMC curve because the matching score is often exactly zero (when the compared fingerprints do not match some conditions). This causes some 
Table 11: TPR percentage for FVC databases

\begin{tabular}{|c|c|c|c|c|c|c|c|c|c|c|c|c|}
\hline Database & Bozorth3 & Jiang & Ratha & Tan & Tico & Deng & Qi & Chen & Chikkerur & Feng & $\mathrm{MCC}$ & $\mathrm{MCC}+\mathrm{L} 1$ \\
\hline FVC2000_db1a & 87.857 & 78.571 & 37.714 & 50.143 & 10.857 & 86.286 & 49.286 & 29.286 & 3.000 & 28.571 & 85.286 & 43.429 \\
\hline FVC2000_db2a & 82.857 & 71.714 & 42.857 & 45.286 & 5.143 & 85.286 & 49.000 & 33.286 & 4.429 & 12.714 & 81.571 & 50.714 \\
\hline FVC2000_db3a & 63.143 & 45.286 & 26.571 & 17.857 & 2.714 & 72.571 & 10.714 & 33.143 & 2.286 & 2.143 & 54.000 & 50.714 \\
\hline FVC2000_db4a & 88.571 & 82.429 & 24.857 & 38.429 & 8.429 & 70.286 & 10.000 & 51.143 & 3.429 & 7.429 & 91.571 & 52.000 \\
\hline FVC2002_db1a & 77.714 & 75.857 & 53.143 & 49.571 & 8.000 & 78.286 & 42.571 & 35.143 & 3.571 & 47.286 & 77.429 & 44.000 \\
\hline FVC2002_db2a & 80.143 & 81.429 & 69.286 & 52.000 & 6.571 & 83.143 & 54.286 & 47.571 & 4.000 & 56.429 & 80.429 & 58.143 \\
\hline FVC2002_db3a & 63.571 & 41.000 & 18.714 & 20.286 & 12.286 & 60.857 & 25.000 & 13.286 & 1.857 & 8.571 & 50.143 & 21.286 \\
\hline FVC2002_db4a & 48.000 & 44.571 & 28.714 & 31.000 & 2.143 & 50.857 & 23.857 & 8.714 & 3.143 & 14.000 & 46.857 & 29.000 \\
\hline FVC2004_db1a & 65.286 & 46.714 & 23.143 & 9.000 & 1.429 & 61.857 & 23.857 & 16.143 & 2.571 & 29.143 & 60.857 & 23.143 \\
\hline FVC2004_db2a & 64.714 & 46.429 & 20.286 & 11.714 & 3.000 & 63.000 & 25.571 & 12.286 & 2.571 & 5.000 & 58.429 & 21.571 \\
\hline FVC2004_db3a & 86.714 & 65.571 & 16.000 & 29.286 & 6.714 & 83.857 & 19.429 & 61.429 & 2.571 & 1.000 & 74.429 & 57.286 \\
\hline FVC2004_db4a & 37.286 & 34.143 & 21.000 & 26.429 & 1.857 & 29.000 & 17.571 & 4.714 & 3.286 & 9.857 & 32.714 & 25.429 \\
\hline
\end{tabular}

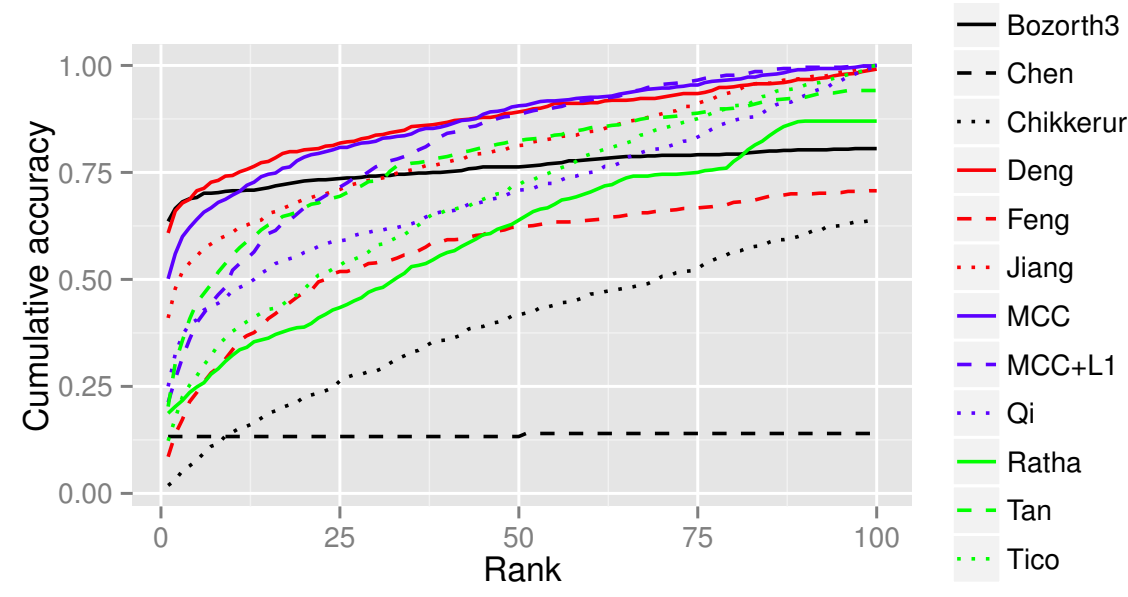

Figure 2: CMC curves for FVC2002_db3a

genuine scores to be the lowest ones in the fingerprint database, and therefore the rank necessary to ensure a certain identification accuracy is greatly increased.

Table 12 displays the results of the statistical tests.

In general, the identification results of these algorithms are similar to the ones obtained for verification, and their behavior remains the same.

To conclude the study, Figure 3 outlines two directed-graphs for verification and identification statistical results respectively. Each method is represented as a vertex, and the edges connect two methods in which the Holm test has detected significant differences. Specifically, in Figure 3a, those methods that receive an arrow are outperformed by the linked algorithm in terms of EER, whereas in Figure 3b, we focus on the TPR measure. A Thick line means that a method statistically outperform another considering all the verification or identification measures. To simplify the graphs, the methods with identical differences with the others have been grouped in the same nodes. 
Table 12: Statistical tests for the identification measures over the FVC databases

\begin{tabular}{|l|r|r|r|}
\hline Algorithm & TPR & R100 & ZeroR \\
\hline Bozorth3 & $\mathbf{1 . 6 6 7}$ & 8.875 & 7.375 \\
Jiang & 3.667 & 3.458 & $\mathbf{4 . 0 8 3}$ \\
Ratha & 7.208 & 8.875 & 7.375 \\
Tan & 7.083 & 8.292 & 7.375 \\
Tico & 10.917 & 5.625 & 6.917 \\
Deng & 1.917 & 6.333 & 6.792 \\
Qi & 7.250 & 6.167 & 7.000 \\
Chen & 8.417 & 8.875 & 7.375 \\
Chikkerur & 11.583 & 8.875 & 7.375 \\
Feng & 9.417 & 8.875 & 7.375 \\
MCC & 2.833 & 1.958 & 4.625 \\
MCC+L1 & 6.042 & $\mathbf{1 . 7 9 2}$ & 4.333 \\
\hline Friedman P-value & $5.652 \mathrm{e}-11$ & $5.652 \mathrm{e}-11$ & 0.089 \\
\hline
\end{tabular}

The figure ratifies the analysis of the accuracy measures: Bozorth3, MCC, Jiang and Deng are the most accurate algorithms for the FVC databases, with statistically significant differences with respect to the other methods.

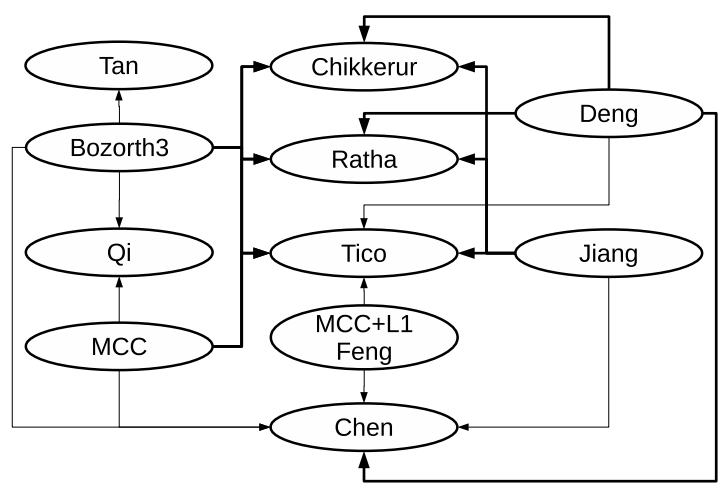

(a) Verification

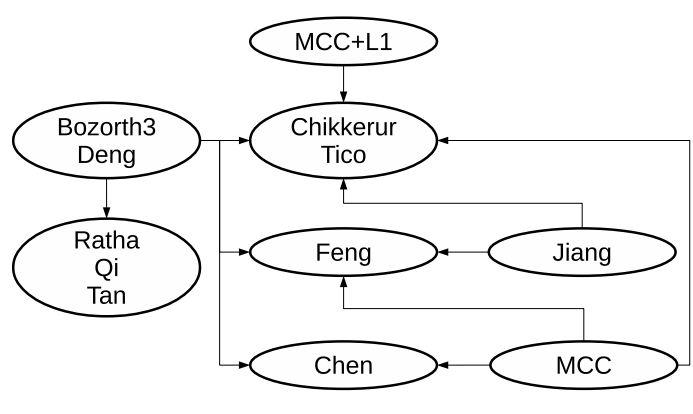

(b) Identification

Figure 3: Significant differences among the tested methods

\subsection{Analysis and Empirical Results on Captured Databases}

In the preceding section, the algorithms of Bozorth3 [165], Jiang [81], Deng [46] and MCC [26] were highlighted as the most accurate for the FVC databases, as they are statistically better than other methods both for verification and identification. This section performs a deeper study upon the four captured databases described, focusing on these four algorithms. 


\subsubsection{Verification}

Table 13 presents the results obtained in terms of EER, FMR100 and FMR1000. Figure 4 displays the ROC curves.

Table 13: Verification performance measures (in percentages)

\begin{tabular}{|l|rrr|rrrrrrrr|rr|r|}
\hline & \multicolumn{4}{|c|}{ EER } & \multicolumn{4}{c|}{ FMR100 } & \multicolumn{4}{c|}{ FMR1000 } \\
Database & Bozorth3 & Jiang & Deng & MCC & Bozorth3 & Jiang & Deng & MCC & Bozorth3 & Jiang & Deng & MCC \\
\hline DB1 & $\mathbf{2 . 7 6 3}$ & 6.292 & 4.288 & 3.448 & $\mathbf{4 . 8 5 2}$ & 15.092 & 9.337 & 6.908 & $\mathbf{1 1 . 1 4 4}$ & 29.223 & 22.733 & 15.638 \\
DB2 & 0.686 & 3.712 & 3.393 & $\mathbf{0 . 3 5 0}$ & 0.617 & 6.546 & 6.056 & $\mathbf{0 . 1 8 0}$ & 1.219 & 14.131 & 15.309 & $\mathbf{0 . 6 2 3}$ \\
DB3 & 0.839 & 2.518 & 1.018 & $\mathbf{0 . 4 1 4}$ & 0.779 & 4.013 & 1.025 & $\mathbf{0 . 2 8 0}$ & 2.103 & 9.177 & 2.845 & $\mathbf{0 . 8 8 9}$ \\
DB4 & 0.788 & 2.512 & 0.951 & $\mathbf{0 . 4 4 3}$ & 0.701 & 3.958 & 0.929 & $\mathbf{0 . 3 0 3}$ & 1.951 & 8.806 & 2.624 & $\mathbf{0 . 8 3 4}$ \\
\hline
\end{tabular}

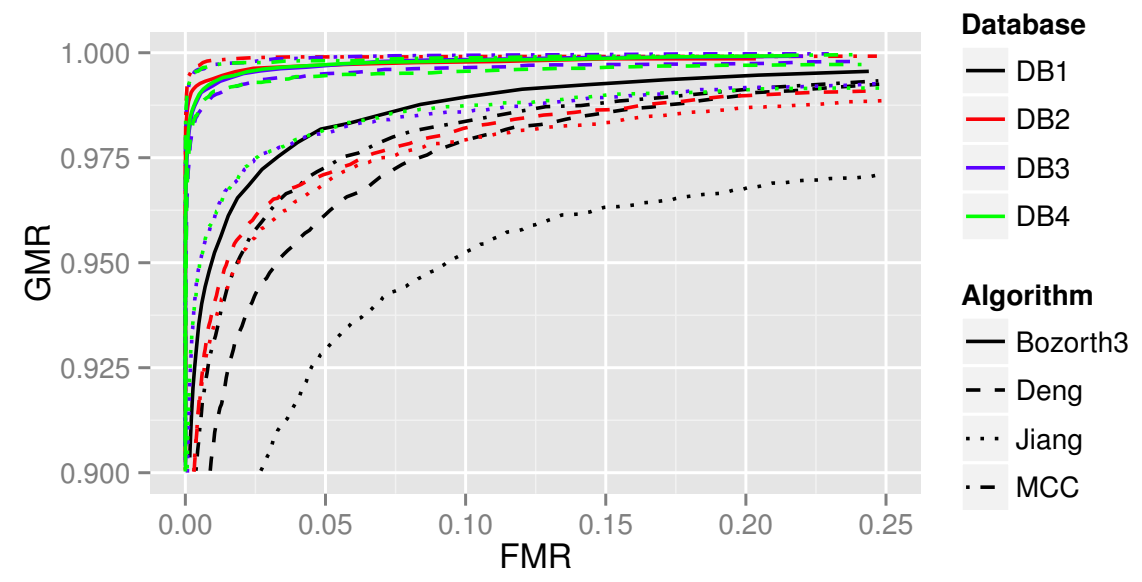

Figure 4: ROC curves for captured databases

Note that the error values for these databases are far better than those obtained for the FVC ones, which are designed for test purposes and whose quality is deliberately bad.

In this case, MCC obtains the best results for all measures and databases except DB1, in which Bozorth3 is better, and the ROC curves follow the same behavior. Jiang gets the worst values among the three tested algorithms.

MCC and Bozorth3 only use the basic minutiae information to build their local structures, while Deng takes into account texture information and some minutiae peculiarities such as the ridge count and the type. Therefore, the fact that Deng is able to obtain good results with the FVC databases - even though it is outperformed by MCC and Bozorth3 for the captured ones - suggests that the texture is less affected than the minutiae in the FVC bad quality images.

It is also noteworthy that Jiang and Deng perform better with the DB3 and DB4 databases (plain fingerprints), while Bozorth3 excels on DB1 (swipe fingerprints), and MCC obtains better results with DB2 (rolled fingerprints). This could happen due to the convex hull computation carried out by MCC, which filters the minutiae on the borders of the fingerprint. Bozorth3, Deng and Jiang do not carry out any special treatment on those areas, 
which are more prone to errors. In all cases, the DB1 database (captured with a narrow swipe sensor) is the most difficult one for the verification.

As for the computing times, we observe the same behavior as with the FVC databases (Table 14). Jiang is the fastest algorithm, followed by Bozorth3, MCC and Deng, which involve more complex consolidations and more information.

Table 14: Average matching times (in milliseconds)

\begin{tabular}{|l|rrrr|}
\hline Database & Bozorth3 & Jiang & Deng & MCC \\
\hline DB1 & 3.679 & $\mathbf{0 . 4 6 9}$ & 11.178 & 6.061 \\
DB2 & 12.076 & $\mathbf{7 . 5 0 1}$ & 175.132 & 64.826 \\
DB3 & 3.227 & $\mathbf{0 . 4 1 5}$ & 9.057 & 5.884 \\
DB4 & 3.184 & $\mathbf{0 . 4 2 3}$ & 9.054 & 5.797 \\
\hline
\end{tabular}

\subsubsection{Identification}

To conclude this study, Table 15 and Figure 5 present the identification performance measures and the CMC for the four tested algorithms over the four captured databases.

Table 15: Identification performance values (1228 templates)

\begin{tabular}{|c|c|c|c|c|c|c|c|c|c|c|c|c|}
\hline \multirow[b]{2}{*}{ Database } & \multicolumn{4}{|c|}{ R100 } & \multicolumn{4}{|c|}{ ZeroR } & \multicolumn{4}{|c|}{ TPR } \\
\hline & Bozorth3 & Jiang & Deng & MCC & Bozorth3 & Jiang & Deng & $\mathrm{MCC}$ & Bozorth3 & Jiang & Deng & $\mathrm{MCC}$ \\
\hline DB1 & 1228 & 866 & 147 & 237 & 1228 & 1228 & 1228 & 1169 & $90.264 \%$ & $69.942 \%$ & $85.125 \%$ & $84.057 \%$ \\
\hline DB2 & 1 & 297 & 121 & 1 & 1228 & 1228 & 1220 & 1202 & $99.077 \%$ & $87.559 \%$ & $93.838 \%$ & $99.222 \%$ \\
\hline DB3 & 1 & 172 & 6 & 1 & 1228 & 1224 & 1228 & 1027 & $99.050 \%$ & $90.771 \%$ & $98.082 \%$ & $99.285 \%$ \\
\hline DB4 & 1 & 118 & 4 & 1 & 1228 & 1228 & 1228 & 1228 & $99.168 \%$ & $90.879 \%$ & $98.172 \%$ & $99.358 \%$ \\
\hline
\end{tabular}

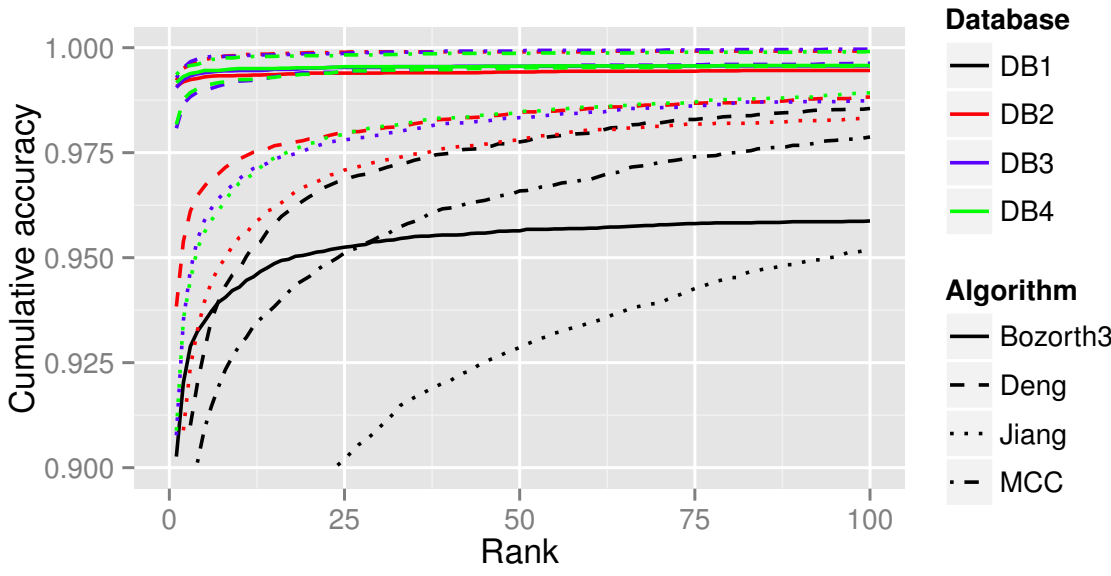

Figure 5: CMC curves for the captured databases

Again, MCC highlights as the most accurate algorithm, except for the DB1 database, for which Deng obtains better R100 and Bozorth obtains better TPR. The CMC curves 
illustrate these results, showing that for low ranks, Bozorth3 performs better than MCC and Deng over DB1. As the rank increases, the cumulative accuracy of Deng increases too. This result contrasts with the verification analysis, which stated that both Bozorth3 and MCC outperform Deng for all databases.

The explanation of this fact is that the verification performance measures are calculated considering a fixed score. That is, each point of the ROC curve plots the FMR and FNMR obtained with a certain score. However, the rank is independent of the numerical value of the scores: it only takes into account their order. The different behavior of ROC and CMC means that the score variability over these databases is higher for Deng than for MCC and Bozorth3. This means that, given an input fingerprint, Deng can ensure with a high confidence that the genuine score is higher than the impostor ones; however, it does not ensure with the same confidence that the genuine and impostor scores of all fingerprints can be separated by a certain fixed threshold.

Otherwise, the relative performance of the databases is maintained: the swipe sensor provides fingerprints that are more difficult to recognize, as well as the DB2 sensor for rolled fingerprints.

\section{Conclusions}

In this paper, we have compiled the most relevant work in the scientific literature about fingerprint local minutiae-based matching. We have described the background in the field, including some references about global matching and feature extraction techniques. Then, we have studied the main properties of the local matching algorithms, as well as the information they are based on, distinguishing between five different aspects: topology of local structure, type of consolidation, usage of additional features, minutiae peculiarities and parameter learning. Using all this information we have built a taxonomy of more than 80 local minutiae matching methods.

In order to complete the study, we have designed and implemented an experimental framework using two sets of databases: 12 from the FVC competitions, which are publicly available, and 4 databases captured by the authors' research groups. The study analyzes the results of 12 of the studied matchers, both in terms of verification and identification performance measures.

After the work realized in this paper, the following conclusions can be drawn:

- Fingerprint matching is a very active field, with dozens of proposed matching methods.

- The obtained results reveal big differences in the accuracy of the matchers, highlighting some of them as more precise than the others.

- The best performing algorithms do not share any special characteristics, although none of them uses any fingerprint features apart in addition to the minutiae coordinates, angle, type and ridge count.

- Furthermore, it has been seen that for different databases, different matchers may be the most accurate. An especially revealing result is that there is a big difference 
between processing rolled, plain and swipe fingerprints, as the different number of minutiae and the presence or not of minutiae on the borders affects the behavior of the matchers.

- This states that some of the different approaches to design matching algorithms are equally valid, and depend on the particular fingerprints.

- There is also a big difference in the computational complexity of the methods: the fastest methods are more suitable for systems with very large fingerprint databases.

- This paper can help nonexperts to choose an appropriate matching algorithm that suits their particular problem.

- It can also help other researchers in the field to develop new matching methods, using the components and properties described in this paper.

In our opinion, the specialized literature contains lots of ideas related to minutiae fingerprint matching, some of them are quite similar and even it may be possible to find overlap among them. Most of the fingerprint matching approaches introduced in the last four decades are minutiae based. One of the reasons to expect minutiae-based algorithms to perform well is the sheer amount of research done on this approach. Original ideas are those which have served as inspiration of the rest of the matching methods. The majority of them were analyzed in this paper with empirical studies, trying to fix one of the main problems observed in this respect in the literature: almost all the proposals were compared under different configurations and without a standard. However, this task is very tedious due to the fact that the papers do not provide all the details to achieve a perfect implementation of the idea presented, especially the information related to the values of the parameters employed.

In the theoretical slope, we realize that the usage of isolated minutiae for matching, although is enough to achieve competitive performance, falls short in more complex scenarios. This is the reason that justifies the fact of real life implementations of fingerprint systems that fuse fingerprints with other traits or employ double fingerprint inputs. The worldwide large scale deployment of fingerprint systems demands a new generation of accurate and highly interoperable algorithms; therefore the development of minutiae-only matching algorithms will not be abandoned for a long time.

In the practical slope, the experiments have shown that none of the features established in the taxonomy can be considered as better than the others, and that the matching algorithms work as a whole. The same algorithms have also been proven to perform differently in different databases. Therefore, all the local structures, consolidations and features described in the taxonomy can be useful for future developments, as the key of an accurate matching algorithm is an adequate use of these parts and not the parts themselves. It has also been noted that the difference in the identification time can be huge depending on the used methods, especially for rolled fingerprints. When time is a limited resource, care must be taken on choosing local structures and consolidations that are at most linear or quadratic with respect to the number of minutiae. 
As future research directions, we particularize the following ones:

- Biometric Fusion: the main advantage of fusion in the context of biometrics is an improvement in the overall matching accuracy. This is commonly known as multifactor authentication and is considered more secure than using fingerprints alone as these other factors have some of their own strengths. Combining fingerprints with other biometric traits offers several advantages, such as the improvement of the universality or the problems caused by the acquisition of poor quality images due to external factors.

- Indexing and Big Data: as we mention in Section 4.2, the indexing is particularly useful when large volumes of fingerprints are stored every day. Identification task in large data bases could become in a real challenge for obtaining quick responses for each query. The employment of Big Data solutions to fingerprint matching and indexing is incoming in the near future.

- High quality images: in certain applications, it is possible to acquire high resolution images in which at the very-fine level, intra-ridge details can be detected. These include width, shape, curvature, edge contours of ridges as well as other permanent details such as dots and incipient ridges. One of the most important fine-level details is the finger sweat pores, whose positions and shapes are considered highly distinctive.

\section{Acknowledgements}

This work was supported by the Research Projects CAB(CDTI), TIN2011-28488, and TIN2013-40765-P. D. Peralta holds an FPU scholarship from the Spanish Ministry of Education and Science (FPU12/04902).

\section{References}

[1] C. Arcelli, G.S. di Baja, A width-independent fast thinning algorithm., IEEE Transactions on Pattern Analysis and Machine Intelligence 7 (1985) 463-474.

[2] A. Balti, M. Sayadi, F. Fnaiech, Improved features for fingerprint identification, in: Proceedings of the Mediterranean Electrotechnical Conference - MELECON, 2012, pp. 878-883.

[3] S. Bayram, H.T. Sencar, N. Memon, Efficient sensor fingerprint matching through fingerprint binarization, IEEE Transactions on Information Forensics and Security 7 (2012) 1404-1413.

[4] A.M. Bazen, S.H. Gerez, Systematic methods for the computation of the directional fields and singular points of fingerprints., IEEE Transations on Pattern Analysis and Machine Intelligence 24 (2002) 905919.

[5] A.M. Bazen, S.H. Gerez, Fingerprint matching by thin-plate spline modelling of elastic deformations., Pattern Recognition 36 (2003) 1859-1867.

[6] A. Bengueddoudj, S. Akrouf, F. Belhadj, D. Nada, Improving fingerprint minutiae matching using local and global structures, in: 8th International Workshop on Systems, Signal Processing and Their Applications, WoSSPA 2013, 2013, pp. 279-282.

[7] F. Benhammadi, M.N. Amirouche, H. Hentous, K. Bey-Beghdad, M. Aissani, Fingerprint matching from minutiae texture maps., Pattern Recognition 40 (2007) 189-197. 
[8] F. Benhammadi, K.B. Beghdad, H. Hentous, Fingerprint verification based on core point neighbourhoods minutiae, in: AICCSA 08 - 6th IEEE/ACS International Conference on Computer Systems and Applications, 2008, pp. 530-536.

[9] F. Benhammadi, K.B. Bey, Embedded fingerprint matching on smart card, International Journal of Pattern Recognition and Artificial Intelligence 27 (2013).

[10] B. Bhanu, X. Tan, Fingerprint indexing based on novel features of minutiae triplets, IEEE Transactions on Pattern Analysis and Machine Intelligence 25 (2003) 616-622.

[11] P. Bhowmick, B.B. Bhattacharya, Approximate fingerprint matching using kd-tree., in: International Conference on Pattern Recognition (ICPR (1)), 2004, pp. 544-s547.

[12] Z. Bian, D. Zhang, W. Shu, Knowledge-based fingerprint post-processing, International Journal of Pattern Recognition and Artificial Intelligence 16 (2002) 53-67.

[13] S. Bistarelli, F. Santini, A. Vaccarelli, An asymmetric fingerprint matching algorithm for java card tm, Pattern Analysis and Applications (2006) 359-376.

[14] J. Bohn, V. Despigel, Fingerprint skeleton matching based on local descriptor, in: IEEE 3rd International Conference on Biometrics: Theory, Applications and Systems, BTAS 2009, 2009.

[15] J. Bringer, V. Despiegel, Binary feature vector fingerprint representation from minutiae vicinities, in: IEEE 4th International Conference on Biometrics: Theory, Applications and Systems, BTAS 2010, 2010.

[16] M.J. Burge, K.W. Bowyer, Handbook of Iris Recognition, Springer Publishing Company, Incorporated, 2013.

[17] K. Cao, X. Yang, X. Chen, X. Tao, Y. Zang, J. Liang, J. Tian, Minutia handedness: A novel global feature for minutiae-based fingerprint matching., Pattern Recognition Letters 33 (2012) 1411-1421.

[18] K. Cao, X. Yang, X. Chen, Y. Zang, J. Liang, J. Tian, A novel ant colony optimization algorithm for large-distorted fingerprint matching., Pattern Recognition 45 (2012) 151-161.

[19] K. Cao, X. Yang, X. Tao, P. Li, Y. Zang, J. Tian, Combining features for distorted fingerprint matching, Journal of Network and Computer Applications 33 (2010) 258-267.

[20] K. Cao, X. Yang, X. Tao, Y. Zhang, J. Tian, A novel matching algorithm for distorted fingerprints based on penalized quadratic model, in: IEEE 3rd International Conference on Biometrics: Theory, Applications and Systems, BTAS 2009, 2009.

[21] K. Cao, X. Yang, J. Tian, Y. Zhang, P. Li, X. Tao, Fingerprint matching based on neighboring information and penalized logistic regression, in: International Conference on Advances in Biometrics (ICB), volume 5558 of Lecture Notes in Computer Science, 2009, pp. 617-626.

[22] R. Cappelli, Fast and accurate fingerprint indexing based on ridge orientation and frequency., IEEE Transactions on Systems, Man, and Cybernetics, Part B 41 (2011) 1511-1521.

[23] R. Cappelli, M. Ferrara, A fingerprint retrieval system based on level-1 and level-2 features., Expert Systems with Applications 39 (2012) 10465-10478.

[24] R. Cappelli, M. Ferrara, D. Maio, Candidate list reduction based on the analysis of fingerprint indexing scores., IEEE Transactions on Information Forensics and Security 6 (2011) 1160-1164.

[25] R. Cappelli, M. Ferrara, D. Maio, A fast and accurate palmprint recognition system based on minutiae, IEEE Transactions on Systems, Man, and Cybernetics, Part B: Cybernetics 42 (2012) 956-962.

[26] R. Cappelli, M. Ferrara, D. Maltoni, Minutia cylinder-code: A new representation and matching technique for fingerprint recognition., IEEE Transactions on Pattern Analysis and Machine Intelligence 32 (2010) 2128-2141.

[27] R. Cappelli, A. Lumini, D. Maio, D. Maltoni, Fingerprint classification by directional image partitioning., IEEE Transactions on Pattern Analysis and Machine Intelligence 21 (1999) 402-421.

[28] C. Carvalho, H. Yehia, Fingerprint alignment using line segments., in: International Conference on Biometric Authentication (ICBA), volume 3072 of Lecture Notes in Computer Science, Springer, 2004, pp. $380-387$.

[29] J.H. Cha, H. Jang, G.Y. Kim, H.I. Choi, Fingerprint matching based on linking information structure of minutiae., in: International Conference on Computational Science and its Applications (ICCSA (1)), volume 3043 of Lecture Notes in Computer Science, 2004, pp. 41-48. 
[30] K.C. Chan, Y.S. Moon, P.S. Cheng, Fast fingerprint verification using subregions of fingerprint images, IEEE Transactions on Circuits Systems and Video Technology 14 (2004) 95-101.

[31] A.C. Chau, C.P. Soto, Hybrid algorithm for fingerprint matching using delaunay triangulation and local binary patterns, in: 16th Iberoamerican Congress on Progress in Pattern Recognition, Image Analysis, Computer Vision, and Applications (CIARP), volume 7042 of Lecture Notes in Computer Science, 2011, pp. 692-700.

[32] F. Chen, X. Huang, J. Zhou, Hierarchical minutiae matching for fingerprint and palmprint identification, IEEE Transactions on Image Processing 22 (2013) 4964-4971.

[33] K. Chen, A. Hu, Fingerprint matching using texture feature extracted from minutiae neighborhood, in: Proceedings - 4th International Conference on Computational Intelligence and Communication Networks, CICN 2012, 2012, pp. 322-326.

[34] X. Chen, J. Tian, J. Cheng, X. Yang, Segmentation of fingerprint images using linear classifier., EURASIP Journal of Advanced Signal Processing 4 (2004) 480-494.

[35] X. Chen, J. Tian, X. Yang, A new algorithm for distorted fingerprints matching based on normalized fuzzy similarity measure., IEEE Transactions on Image Processing 15 (2006) 767-776.

[36] X. Chen, J. Tian, X. Yang, Y. Zhang, An algorithm for distorted fingerprint matching based on local triangle feature set, IEEE Transactions on Information Forensics and Security 1 (2006) 169-177.

[37] J. Cheng, J. Tian, H. Chen, Fingerprint minutiae matching with orientation and ridge, in: International Conference on Biometric Authentication (ICBA), 2004, pp. 351-358.

[38] X. Cheng, S. Tulyakov, V. Govindaraju, Minutiae-based matching state model for combinations in fingerprint matching system, in: IEEE Computer Society Conference on Computer Vision and Pattern Recognition Workshops, 2013, pp. 92-97.

[39] S. Chikkerur, A.N. Cartwright, V. Govindaraju, K-plet and coupled BFS: A graph based fingerprint representation and matching algorithm., in: International Conference on Biometrics (ICB), volume 3832 of Lecture Notes in Computer Science, 2006, pp. 309-315.

[40] S. Chikkerur, S. Pankanti, A. Jea, N.K. Ratha, R.M. Bolle, Fingerprint representation using localized texture features., in: International Conference on Pattern Recognition (ICPR (4)), 2006, pp. 521-524.

[41] S. Chikkerur, N.K. Ratha, Impact of singular point detection on fingerprint matching performance., in: workshop on Automatic Identification Advanced Technologies, 2005, pp. 207-212.

[42] H. Choi, K. Choi, J. Kim, Fingerprint matching incorporating ridge features with minutiae., IEEE Transactions on Information Forensics and Security 6 (2011) 338-345.

[43] V. Conti, G. Vitello, F. Sorbello, S. Vitabile, An advanced technique for user identification using partial fingerprint, in: Proceedings of the 7th International Conference on Complex, Intelligent, and Software Intensive Systems, CISIS 2013, 2013, pp. 236-242.

[44] J. Dai, J. Feng, J. Zhou, Robust and efficient ridge-based palmprint matching, IEEE Transactions on Pattern Analysis and Machine Intelligence 34 (2012) 1618-1632.

[45] J. Demšar, Statistical comparisons of classifiers over multiple data sets, The Journal of Machine Learning Research 7 (2006) 1-30.

[46] H. Deng, Q. Huo, Minutiae matching based fingerprint verification using delaunay triangulation and aligned-edge-guided triangle matching, in: Proceedings of the 5th International Conference on Audioand Video-Based Biometric Person Authentication, AVBPA, 2005, pp. 270-278.

[47] K.C. Fan, C.W. Liu, Y.K. Wang, A randomized approach with geometric constraints to fingerprint verification., Pattern Recognition 33 (2000) 1793-1803.

[48] G. Fang, S.N. Srihari, H. Srinivasan, P. Phatak, Use of ridge points in partial fingerprint matching, in: SPIE: Biometric Technology for Human Identification IV, 2007.

[49] J. Feng, Combining minutiae descriptors for fingerprint matching., Pattern Recognition 41 (2008) $342-352$.

[50] J. Feng, Z. Ouyang, A. Cai, Fingerprint matching using ridges, Pattern Recognition 39 (2006) 21312140.

[51] M. Ferrara, D. Maltoni, R. Cappelli, Noninvertible minutia cylinder-code representation, IEEE Transactions on Information Forensics and Security 7 (2012) 1727-1737. 
[52] M. Friedman, The use of ranks to avoid the assumption of normality implicit in the analysis of variance, Journal of the American Statistical Association 32 (1937) 675-701.

[53] Z. Gao, X. You, L. Zhou, W. Zeng, A novel matching technique for fingerprint recognition by graphical structures, in: International Conference on Wavelet Analysis and Pattern Recognition, 2011, pp. 7782.

[54] S. García, F. Herrera, An extension on "statistical comparisons of classifiers over multiple data sets" for all pairwise comparisons, Journal of Machine Learning Research 9 (2008) 2677-2694.

[55] R. Garg, S. Rane, A keypoint descriptor for alignment-free fingerprint matching, in: ICASSP, IEEE International Conference on Acoustics, Speech and Signal Processing - Proceedings, 2013, pp. 29942998.

[56] J. Gu, J. Zhou, C. Yang, Fingerprint recognition by combining global structure and local cues, IEEE Transactions on Image Processing 15 (2006) 1952-1964.

[57] H. Guesmi, H. Trichili, A.M. Alimi, B. Solaiman, Fingerprint verification system based on curvelet transform and possibility theory, Multimedia Tools and Applications, in press. DOI: 10.1007/s11042013-1785-1 (2014) 1-20.

[58] Z. Guo, R.W. Hall, Parallel thinning with two-subiteration algorithms., Communications of the ACM 32 (1989) 359-373. Corrigendum: CACM 32(6): 759 (1989).

[59] P.D. Gutiérrez, M. Lastra, F. Herrera, J.M. Benitez, A high performance fingerprint matching system for large databases based on GPU, IEEE Transactions on Information Forensics and Security 9 (2014) $62-71$.

[60] H. Hasan, S.A. Kareem, Fingerprint image enhancement and recognition algorithms: a survey, Neural Computing and Applications 23 (2013) 1605-1610.

[61] X. He, J. Tian, L. Li, Y. He, X. Yang, Modeling and analysis of local comprehensive minutia relation for fingerprint matching, IEEE Transactions on Systems, Man, and Cybernetics, Part B 37 (2007) 1204-1211.

[62] Y. He, J. Tian, L. Li, H. Chen, X. Yang, Fingerprint matching based on global comprehensive similarity, IEEE Transactions on Pattern Analysis and Machine Intelligence 28 (2006) 850-862.

[63] Y. He, J. Tian, X. Luo, T. Zhang, Image enhancement and minutiae matching in fingerprint verification, Pattern Recognition Letters 24 (2003) 1349-1360.

[64] L. Hong, A. Jain, Integrating faces and fingerprints for personal identification, IEEE Transactions on Pattern Analysis and Machine Intelligence 20 (1998) 1295-1307.

[65] L. Hong, Y. Wan, A. Jain, Fingerprint image enhancement: Algorithm and performance evaluation, IEEE Transactions on Pattern Analysis and Machine Intelligence 20 (1998) 777-789.

[66] K. Ito, H. Nakajima, K. Kobayashi, T. Aoki, T. Higuchi, A fingerprint matching algorithm using phase-only correlation, IEICE Transactions on Fundamentals of Electronics, Communications and Computer Sciences E87-A (2004) 682-691.

[67] M.H. Izadi, L. Mirmohamadsadeghi, A. Drygajlo, Introduction of cylinder quality measure into minutia cylinder-code based fingerprint matching, in: 2012 IEEE 5th International Conference on Biometrics: Theory, Applications and Systems, BTAS 2012, 2012, pp. 353-358.

[68] A.K. Jain, Y. Chen, M. Demirkus, Pores and ridges: High-resolution fingerprint matching using level 3 features, IEEE Transactions on Pattern Analysis and Machine Intelligence 29 (2007) 15-27.

[69] A.K. Jain, J. Feng, Latent palmprint matching, IEEE Transactions on Pattern Analysis and Machine Intelligence 31 (2009) 1032-1047.

[70] A.K. Jain, J. Feng, Latent fingerprint matching, IEEE Transactions on Pattern Analysis and Machine Intelligence 33 (2011) 88-100.

[71] A.K. Jain, J. Feng, K. Nandakumar, Fingerprint matching, IEEE Computer 43 (2010) 36-44.

[72] A.K. Jain, L. Hong, R.M. Bolle, On-Line Fingerprint Verification, IEEE Transactions on Pattern Analysis and Machine Intelligence 19 (1997) 302-314.

[73] A.K. Jain, S. Prabhakar, S. Chen, Combining multiple matchers for a high security fingerprint verification system, Pattern Recognition Letters 20 (1999) 1371-1379.

[74] A.K. Jain, S. Prabhakar, L. Hong, A multichannel approach to fingerprint classification., IEEE Trans- 
actions on Pattern Analysis and Machine Intelligence 21 (1999) 348-359.

[75] A.K. Jain, S. Prabhakar, L. Hong, S. Pankanti, Filterbank-based fingerprint matching, IEEE Transactions on Image Proccessing 9 (2000) 846-859.

[76] A.K. Jain, A.A. Ross, K. Nandakumar, Introduction to Biometrics, Springer Publishing Company, Incorporated, 2011.

[77] T.Y. Jea, V. Govindaraju, A minutia-based partial fingerprint recognition system, Pattern Recognition 38 (2005) 1672-1684.

[78] J. Jia, L. Cai, P. Lu, X. Liu, Fingerprint matching based on weighting method and the svm., Neurocomputing 70 (2007) 849-858.

[79] R.M. Jiang, D. Crookes, Fpga-based minutia matching for biometric fingerprint image database retrieval, Journal of Real-Time Image Processing 3 (2008) 177-182.

[80] X. Jiang, M. Liu, A.C. Kot, Fingerprint retrieval for identification., IEEE Transactions on Information Forensics and Security 1 (2006) 532-542.

[81] X. Jiang, W.Y. Yau, Fingerprint minutiae matching based on the local and global structures, in: International Conference on Pattern Recognition (ICPR), 2000, pp. 6038-6041.

[82] X. Jiang, X. You, Y. Yuan, M. Gong, A method using long digital straight segments for fingerprint recognition, Neurocomputing 77 (2012) 28-35.

[83] Y. Jie, Y. Yi fang, Z. Renjie, S. Qifa, Fingerprint minutiae matching algorithm for real time system, Pattern Recognition 39 (2006) 143-146.

[84] A.T.B. Jin, D.N.C. Ling, O.T. Song, An efficient fingerprint verification system using integrated wavelet and fourier-mellin invariant transform., Image and Vision Computing 22 (2004) 503-513.

[85] K. Karu, A.K. Jain, Fingerprint classification, Pattern Recognition 29 (1996) 389-404.

[86] M. Khalil, D. Muhammad, M. Khan, K. Alghathbar, Singular points detection using fingerprint orientation field reliability., International Journal of Physical Sciences 5 (2010) 352-357.

[87] U.M. Khan, S.A. Khan, N. Ejaz, R.U. Rehman, A fingerprint verification system using minutiae and wavelet based features, in: 2009 International Conference on Emerging Technologies, ICET 2009, 2009, pp. 291-296.

[88] H. Khazaei, A. Mohades, Fingerprint matching algorithm based on voronoi diagram, in: Proceedings - The International Conference on Computational Sciences and its Applications, ICCSA 2008, 2008, pp. 433-440.

[89] A. Kisel, A. Kochetkov, J. Kranauskas, Fingerprint minutiae matching without global alignment using local structures, Informatica 19 (2008) 31-44.

[90] Z.M. Kovács-Vajna, A fingerprint verification system based on triangular matching and dynamic time warping, IEEE Transactions on Pattern Analysis and Machine Intelligence 22 (2000) 1266-1276.

[91] J.V. Kulkarni, B.D. Patil, R.S. Holambe, Orientation feature for fingerprint matching., Pattern Recognition 39 (2006) 1551-1554.

[92] R. Kumar, B.R.D. Vikram, Fingerprint matching using multi-dimensional ann., Engineering Applications of Artificial Intelligence 23 (2010) 222-228.

[93] D. Kwon, I.D. Yun, D.H. Kim, S.U. Lee, Fingerprint matching method using minutiae clustering and warping., in: International Conference on Pattern Recognition (ICPR (4)), 2006, pp. 525-528.

[94] D. Lee, K. Choi, J. Kim, A robust fingerprint matching algorithm using local alignment., in: International Conference on Pattern Recognition (ICPR (3)), 2002, pp. 803-806.

[95] S. Lee, H. seung Choi, K. Choi, J. Kim, Fingerprint-quality index using gradient components., IEEE Transactions on Information Forensics and Security 3 (2008) 792-800.

[96] P. Li, X. Yang, Q. Su, Y. Zhang, J. Tian, A novel fingerprint matching algorithm using ridge curvature feature, in: International Conference on Advances in Biometrics (ICB), volume 5558 of Lecture Notes in Computer Science, 2009, pp. 607-616.

[97] S. Li, A. Kot, Fingerprint combination for privacy protection, IEEE Transactions on Information Forensics and Security 8 (2013) 350-360.

[98] X. Liang, T. Asano, Fingerprint matching using minutia polygons, International Conference on Pattern Recognition (ICPR) 1 (2006) 1046-1049. 
[99] X. Liang, A. Bishnu, T. Asano, A robust fingerprint indexing scheme using minutia neighborhood structure and low-order delaunay triangles., IEEE Transactions on Information Forensics and Security 2 (2007) 721-733.

[100] C. Liu, J. Cao, X. Gao, X. Fu, J. Feng, A novel fingerprint matching algorithm using minutiae phase difference feature, in: Proceedings - International Conference on Image Processing, ICIP, 2011, pp. 3201-3204.

[101] C. Liu, T. Xia, H. Li, A hierarchical hough transform for fingerprint matching., in: International Conference on Biometric Authentication (ICBA), volume 3072 of Lecture Notes in Computer Science, Springer, 2004, pp. 373-379.

[102] E. Liu, A.K. Jain, J. Tian, A coarse to fine minutiae-based latent palmprint matching, IEEE Transactions on Pattern Analysis and Machine Intelligence 35 (2013) 2307-2322.

[103] F. Liu, Q. Zhao, D. Zhang, A novel hierarchical fingerprint matching approach, Pattern Recognition 44 (2011) 1604-1613.

[104] L. Liu, T. Jiang, J. Yang, C. Zhu, Fingerprint registration by maximization of mutual information, IEEE Transactions on Image Processing 15 (2006) 1100-1110.

[105] M. Liu, P.T. Yap, Invariant representation of orientation fields for fingerprint indexing, Pattern Recognition 45 (2012) 2532-2542.

[106] X. Luo, J. Tian, Knowledge based fingerprint image enhancement., in: International Conference on Pattern Recognition (ICPR), 2000, pp. 4783-4786.

[107] X. Luo, J. Tian, Y. Wu, A minutia matching algorithm in fingerprint verification., in: International Conference on Pattern Recognition (ICPR), 2000, pp. 4833-4836.

[108] D. Maio, D. Maltoni, Direct gray-scale minutiae detection in fingerprints, IEEE Transactions on Pattern Analysis and Machine Inteligence 19 (1997) 27-40.

[109] D. Maio, D. Maltoni, Ridge-line density estimation in digital images, in: International Conference on Pattern Recognition (ICPR(1)), 1998, pp. 534-538.

[110] D. Maio, D. Maltoni, R. Cappelli, J. Wayman, A. Jain, FVC2000: fingerprint verification competition, Pattern Analysis and Machine Intelligence, IEEE Transactions on 24 (2002) 402-412.

[111] D. Maio, D. Maltoni, R. Cappelli, J. Wayman, A. Jain, FVC2004: Third fingerprint verification competition, Lecture Notes in Computer Science (including subseries Lecture Notes in Artificial Intelligence and Lecture Notes in Bioinformatics) 3072 (2004) 1-7.

[112] D. Maio, D. Maltoni, R. Cappelli, J.L. Wayman, A.K. Jain, FVC2002: Second fingerprint verification competition, in: Pattern Recognition, 2002. Proceedings. 16th International Conference on, volume 3, 2002, pp. 811-814.

[113] D. Maltoni, D. Maio, A.K. Jain, S. Prabhakar, Handbook of Fingerprint Recognition, Springer Publishing Company, Incorporated, 2nd edition, 2009.

[114] K. Mao, G. Wang, C. Yu, Y. Jin, A novel multi-reference points fingerprint matching method, in: International Conference on Advances in Multimedia Modeling (MMM), volume 5371 of Lecture Notes in Computer Science, 2009, pp. 356-366.

[115] K. Mao, G. Wang, G. Yu, A novel fingerprint matching method by excluding elastic distortion, in: International Conference on Database Systems for Advanced Applications (DASFAA), volume 4947 of Lecture Notes in Computer Science, 2008, pp. 348-363.

[116] M.A. Medina-Pérez, M. García-Borroto, A.E. Gutierrez-Rodríguez, L. Altamirano-Robles, Improving fingerprint verification using minutiae triplets, Sensors 12 (2012) 3418-3437.

[117] M.A. Medina-Prez, A. Gutirrez-Rodrguez, M. Garca-Borroto, Improving fingerprint matching using an orientation-based minutia descriptor, in: 14th Iberoamerican Conference on Pattern Recognition: Progress in Pattern Recognition, Image Analysis, Computer Vision, and Applications (CIARP), volume 5856 of Lecture Notes in Computer Science, 2009, pp. 121-128.

[118] P. Meenen, A. Ashrafi, R.R. Adhami, The utilization of a taylor series-based transformation in fingerprint verification., Pattern Recognition Letters 27 (2006) 1606-1618.

[119] P.I. Mistry, C.N. Paunwala, Fusion fingerprint minutiae matching system for personal identification, in: 4th International Conference on Computing, Communications and Networking Technologies, ICCCNT 
2013, 2013.

[120] K.A. Nagaty, An adaptive hybrid energy-based fingerprint matching technique., Image and Vision Computing 23 (2005) 491-500.

[121] K. Nandakumar, Fingerprint matching based on minutiae phase spectrum., in: International Conference on Biometrics (ICB), 2012, pp. 216-221.

[122] L. Nanni, A. Lumini, Local binary patterns for a hybrid fingerprint matcher, Pattern Recognition 41 (2008) 3461-3466.

[123] G.S. Ng, X. Tong, X. Tang, D. Shi, Adjacent orientation vector based fingerprint minutiae matching system, in: International Conference on Pattern Recognition (ICPR (1)), 2004, pp. 528-531.

[124] K. Nilsson, J. Bigun, Localization of corresponding points in fingerprints by complex filtering, Pattern Recognition Letters 24 (2003) 2135-2144.

[125] L. O'Gorman, J.V. Nickerson, An approach to fingerprint filter design., Pattern Recognition 22 (1989) 29-38.

[126] S. Pankanti, S. Prabhakar, A.K. Jain, On the individuality of fingerprints, IEEE Transactions on Pattern Analysis and Machine Intelligence 24 (2001) 1010-1025.

[127] G. Parziale, A. Niel, A fingerprint matching using minutiae triangulation., in: International Conference on Biometric Authentication (ICBA), volume 3072 of Lecture Notes in Computer Science, 2004, pp. 241-248.

[128] A.A. Paulino, J. Feng, A.K. Jain, Latent fingerprint matching using descriptor-based hough transform, IEEE Transactions on Information Forensics and Security 8 (2013) 31-45.

[129] D. Peralta, M. Galar, I. Triguero, O. Miguel-Hurtado, J.M. Benitez, F. Herrera, Minutiae filtering to improve both efficacy and efficiency of fingerprint matching algorithms, Engineering Applications of Artificial Intelligence 32 (2014) $37-53$.

[130] D. Peralta, I. Triguero, R. Sanchez-Reillo, F. Herrera, J.M. Benitez, Fast fingerprint identification for large databases, Pattern Recognition 47 (2014) 588-602.

[131] V.N. Perminov, A.M. Fartukov, Method for fingerprint minutiae matching based on their alignment, Pattern Recognition and Image Analysis 17 (2007) 631-638.

[132] J. Qi, Y. Wang, A robust fingerprint matching method, Pattern Recognition 38 (2005) 1665-1671.

[133] J. Qi, M. Xie, W. Wang, A novel fingerprint matching method using a curvature-based minutia specifier, in: Proceedings - International Conference on Image Processing, ICIP, 2008, pp. 1488-1491.

[134] J. Qi, S. Yang, Y. Wang, Fingerprint matching combining the global orientation field with minutia, Pattern Recognition Letters 26 (2005) 2424-2430.

[135] C.J. Ran, M. Xie, A new fingerprint matching method based on ridge tracing, in: Proceedings of the 2007 International Conference on Wavelet Analysis and Pattern Recognition, ICWAPR '07, volume 3, 2007, pp. 402-407.

[136] N. Ratha, R. Bolle, V. Pandit, V. Vaish, Robust fingerprint authentication using local structural similarity, in: Workshop on Applications of Computer Vision, 2000, pp. 29-34.

[137] N.K. Ratha, S. Chen, A.K. Jain, Adaptive flow orientation-based feature extraction in fingerprint images., Pattern Recognition 28 (1995) 1657-1672.

[138] N.K. Ratha, K. Karu, S. Chen, A.K. Jain, A real-time matching system for large fingerprint databases., IEEE Transactions on Pattern Analysis and Machine Intelligence 18 (1996) 799-813.

[139] K. Rerkrai, V. Areekul, A new reference point for fingerprint recognition., in: International Conference on Image Processing (ICIP), 2000, pp. 499-502.

[140] A. Ross, S.C. Dass, A.K. Jain, A deformable model for fingerprint matching., Pattern Recognition 38 (2005) 95-103.

[141] A. Ross, A.K. Jain, J. Reisman, A hybrid fingerprint matcher., Pattern Recognition 36 (2003) 16611673.

[142] L. Sha, X. Tang, Orientation-improved minutiae for fingerprint matching, in: Proceedings of the Pattern Recognition, 17th International Conference on (ICPR) Volume 4, 2004, pp. 432-435.

[143] L. Sha, F. Zhao, X. Tang, Minutiae-based fingerprint matching using subset combination, in: International Conference on Pattern Recognition (ICPR (4)), 2006, pp. 566-569. 
[144] W. Sheng, G. Howells, M. Fairhurst, F. Deravi, K. Harmer, Consensus fingerprint matching with genetically optimised approach, Pattern Recognition 42 (2009) 1399-1407.

[145] W. Sheng, G. Howells, M.C. Fairhurst, F. Deravi, A memetic fingerprint matching algorithm., IEEE Transactions on Information Forensics and Security 2 (2007) 402-412.

[146] J. Shi, K.Y. Lam, Minucode: A fixed-value representation of fingerprint minutiae for biometric cryptosystem, in: International Conference on Advances in Information Security and Assurance (ISA), volume 5576 of Lecture Notes in Computer Science, 2009, pp. 382-391.

[147] Z. Shi, V. Govindaraju, Robust fingerprint matching using spiral partitioning scheme, in: International Conference on Advances in Biometrics (ICB), volume 5558 of Lecture Notes in Computer Science, 2009, pp. $647-655$.

[148] F. Su, P. Sun, L. Wang, X. Xie, An efficient minutiae-based fingerprint matching algorithm for resource constrained implementation, in: Proceedings - 2010 2nd IEEE International Conference on Network Infrastructure and Digital Content, IC-NIDC 2010, 2010, pp. 214-218.

[149] V.A. Sujan, M.P. Mulqueen, Fingerprint identification using space invariant transforms., Pattern Recognition Letters 23 (2002) 609-619.

[150] X. Tan, B. Bhanu, A robust two step approach for fingerprint identification, Pattern Recognition Letters 24 (2003) 2127-2134.

[151] X. Tan, B. Bhanu, Fingerprint matching by genetic algorithms, Pattern Recognition 39 (2006) 465477.

[152] N.T.H. Thuy, H.X. Huan, N.N. Ky, An efficient method for fingerprint matching based on local point model, in: International Conference on Computing, Management and Telecommunications, ComManTel 2013, 2013, pp. 334-339.

[153] M. Tico, P. Kuosmanen, An algorithm for fingerprint image postprocessing, in: In Proceedings of the Thirty-Fourth Asilomar Conference on Signals, Systems and Computers, 2000, pp. 1735-1739.

[154] M. Tico, P. Kuosmanen, Fingerprint matching using an orientation-based minutia descriptor., IEEE Transactions on Pattern Analysis and Machine Intelligence 25 (2003) 1009-1014.

[155] X. Tong, J. Huang, X. Tang, D. Shi, Fingerprint minutiae matching using the adjacent feature vector., Pattern Recognition Letters 26 (2005) 1337-1345.

[156] X. Tong, S. Liu, J. Huang, X. Tang, Local relative location error descriptor-based fingerprint minutiae matching, Pattern Recognition Letters 29 (2008) 286-294.

[157] R. Udupa, G. Garg, P.K. Sharma, Fast and accurate fingerprint verification., in: International Conference on Audio and Video based Biometric Person Authentication (AVBPA), volume 2091 of Lecture Notes in Computer Science, 2001, pp. 192-197.

[158] T. Uz, G. Bebis, A. Erol, S. Prabhakar, Minutiae-based template synthesis and matching for fingerprint authentication, Computer Vision and Image Understanding 113 (2009) 979-992.

[159] K. Venkataramani, B.V.K.V. Kumar, Performance of composite correlation filters in fingerprint verification., Optical Engineering 43 (2004) 1820-1827.

[160] M.A. Wahby-Shalaby, M. Omair Ahmad, A multilevel structural technique for fingerprint representation and matching, Signal Processing 93 (2013) 56-69.

[161] D. Wan, J. Zhou, Fingerprint recognition using model-based density map, IEEE Transactions on Image Processing 15 (2006) 1690-1696.

[162] C. Wang, G. Ding, Z. Zheng, Fingerprint matching combining the adjacent feature with curvature of ridges, in: Proceedings of the World Congress on Intelligent Control and Automation (WCICA), 2008, pp. 6807-6810.

[163] W. Wang, J. Li, W. Chen, Fingerprint minutiae matching based on coordinate system bank and global optimum alignment, in: International Conference on Pattern Recognition (ICPR (4)), 2006, pp. 401-404.

[164] X. Wang, J. Li, Y. Niu, Fingerprint matching using orientationcodes and polylines., Pattern Recognition 40 (2007) 3164-3177.

[165] C.I. Watson, M.D. Garris, E. Tabassi, C.L. Wilson, R.M. Mccabe, S. Janet, K. Ko, User's Guide to NIST Biometric Image Software (NBIS), Technical Report, NIST, 2010. 
[166] H. Wei, M. Guo, Z. Ou, Fingerprint verification based on multistage minutiae matching., in: International Conference on Pattern Recognition (ICPR (2)), 2006, pp. 1058-1061.

[167] H. Wei, D. Liu, A multi-stage fingerprints matching algorithm, in: Proceedings of the 2009 IEEE International Conference on Automation and Logistics, ICAL 2009, 2009, pp. 197-199.

[168] C.L. Wilson, C.I. Watson, E.G. Paek, Effect of resolution and image quality on combined optical and neural network fingerprint matching., Pattern Recognition 33 (2000) 317-331.

[169] X. Xie, F. Su, A. Cai, Ridge-based fingerprint recognition., in: International Conference on Biometrics (ICB), volume 3832 of Lecture Notes in Computer Science, 2006, pp. 273-279.

[170] X. Xie, F. Su, A. Cai, J. Sun, A robust fingerprint minutiae matching algorithm based on the support model., in: International Conference on Biometric Authentication (ICBA), volume 3072 of Lecture Notes in Computer Science, 2004, pp. 316-323.

[171] H. Xu, R.N.J. Veldhuis, T.A.M. Kevenaar, A.H.M. Akkermans, A fast minutiae-based fingerprint recognition system., IEEE Systems Journal 3 (2009) 418-427.

[172] W. Xu, X. Chen, J. Feng, A robust fingerprint matching approach: Growing and fusing of local structures., in: International Conference on Biometrics (ICB), volume 4642 of Lecture Notes in Computer Science, 2007, pp. 134-143.

[173] L. Xuzhou, Y. Fei, A new fingerprint matching algorithm based on minutiae, in: Proceedings of 2009 IEEE International Conference on Communications Technology and Applications, IEEE ICCTA2009, 2009, pp. 869-873.

[174] N. Yager, A. Amin, Fingerprint verification based on minutiae features: a review, Pattern Analysis and Applications 7 (2004) 94-113.

[175] N. Yager, A. Amin, Fingerprint alignment using a two stage optimization., Pattern Recognition Letters 27 (2006) 317-324.

[176] J. Yang, S. Xie, S. Yoon, D. Park, Z. Fang, S. Yang, Fingerprint matching based on extreme learning machine, Neural Computing and Applications 22 (2013) 435-445.

[177] W. Yang, J. Hu, M. Stojmenovic, Ndtc: A novel topology-based fingerprint matching algorithm using n-layer delaunay triangulation net check, in: Proceedings of the 2012 7th IEEE Conference on Industrial Electronics and Applications, ICIEA 2012, 2012, pp. 866-870.

[178] Y. Ying, H. Zhang, X. Yang., A method based on delaunay triangulation for fingerprint matching., in: SPIE Conference on Biometric Technology for Human Identification II, 2005.

[179] K.D. Yu, S. Na, T.Y. Choi, A fingerprint matching algorithm based on radial structure and a structurerewarding scoring strategy., in: International Conference on Audio- and Video-based Biometric Person Authentication (AVBPA), volume 3546 of Lecture Notes in Computer Science, 2005, pp. 656-664.

[180] T.Y. Zhang, C.Y. Suen, A fast parallel algorithm for thinning digital patterns, Communications of the ACM 27 (1984) 236-239.

[181] W. Zhang, Y. Wang, Core-based structure matching algorithm of fingerprint verification, in: International Conference on Pattern Recognition (ICPR (1)), 2002, pp. 70-74.

[182] Y. Zhang, X. Yang, Q. Su, J. Tian, Fingerprint recognition based on combined features., in: International Conference on Biometrics (ICB), volume 4642 of Lecture Notes in Computer Science, 2007, pp. 281-289.

[183] D. Zhao, F. Su, A. Cai, Fingerprint registration using minutia clusters and centroid structure, in: International Conference on Pattern Recognition (ICPR (4)), 2006, pp. 413-416.

[184] F. Zhao, X. Tang, Preprocessing and postprocessing for skeleton-based fingerprint minutiae extraction., Pattern Recognition 40 (2007) 1270-1281.

[185] X. Zhao, X. Zhang, G. Zhao, X. Li, K. Zhang, R. Qian, Triangle matching combined with singular features in fingerprints, in: Proceedings 2011 International Conference on Mechatronic Science, Electric Engineering and Computer, MEC 2011, 2011, pp. 2069-2072.

[186] J.D. Zheng, Y. Gao, M.Z. Zhang, Fingerprint matching algorithm based on similar vector triangle, in: Proceedings of the 2009 2nd International Congress on Image and Signal Processing, CISP'09, 2009.

[187] X. Zheng, Y. Wang, Fingerprint matching based on ridge similarity, in: ICASSP, IEEE International Conference on Acoustics, Speech and Signal Processing - Proceedings, 2008, pp. 1701-1704. 
[188] W.B. Zhong, X.B. Ning, C.J. Wei, A fingerprint matching algorithm based on relative topological relationship among minutiae, in: 2008 IEEE International Conference Neural Networks and Signal Processing, ICNNSP, 2008, pp. 225-228.

[189] E. Zhu, J. Yin, G. Zhang, Fingerprint matching based on global alignment of multiple reference minutiae, Pattern Recognition 38 (2005) 1685-1694. 